\title{
Trade-off between angular resolution and straylight contamination in the PLANCK Low Frequency Instrument ${ }^{\star}$
}

\section{Straylight evaluation}

\author{
C. Burigana ${ }^{1}$, M. Sandri ${ }^{1,2}$, F. Villa ${ }^{1}$, D. Maino ${ }^{3}$, R. Paladini ${ }^{4,5}$, C. Baccigalupi ${ }^{4}$, M. Bersanelli $^{3,6}$, and N. Mandolesi ${ }^{1}$ \\ ${ }^{1}$ CNR - INAF/IASF, Sezione di Bologna, via P. Gobetti, 101, 40129 Bologna, Italy \\ e-mail: [burigana;sandri; villa;mandolesi]@abo.iasf.cnr.it \\ 2 Dipartimento di Astronomia, Università degli Studi di Padova, vicolo dell'Osservatorio, 2, 35100 Padova, Italy \\ 3 Dipartimento di Fisica, Università degli Studi di Milano, via G. Celoria, 16, 20133 Milano, Italy \\ e-mail: Marco.Bersanelli@fisica.unimi.it; davide.maino@mi.infn.it \\ 4 SISSA, International School for Advanced Studies, via Beirut, 2-4, 34014 Trieste, Italy \\ e-mail: bacci@sissa.it \\ 5 CESR, 9 avenue du Colonel Roche, BP 4346, 31028 Toulouse, France \\ e-mail: paladini@cesr.fr \\ ${ }^{6}$ CNR - INAF/IASF, Sezione di Milano, via E. Bassini, 15, 20133 Milano, Italy \\ On behalf of the LFI Consortium
}

Received 3 June 2004 / Accepted 27 July 2004

\begin{abstract}
The last generation of CMB anisotropy experiments operating either from space, like the WMAP and PLANCK satellite, from the atmosphere, such as balloons, or from the ground, like interferometers, make use of complex multi-frequency instruments at the focus of meter class telescopes to allow the joint study of CMB and foreground anisotropies, necessary to achieve an accurate component separation. Between $\sim 70 \mathrm{GHz}$ and $\sim 300 \mathrm{GHz}$, where foreground contamination is minimum, it is extremely important to reach the best trade-off between the improvement of the angular resolution, necessary for measuring the high order acoustic peaks of CMB anisotropy, and the minimization of the straylight contamination mainly due to the Galactic emission. This is one of the most critical systematic effects at large and intermediate angular scales (i.e. at multipoles $\ell$ less than $\approx 100$ ) and consists in unwanted radiation entering the beam at large angles from the direction of the antenna boresight direction. We consider here the 30 and $100 \mathrm{GHz}$ channels of the PLANCK Low Frequency Instrument (LFI). Assuming the nominal PLANCK scanning strategy, we evaluate the straylight contamination introduced by the most relevant Galactic foreground components for a reference set of optical configurations, accurately simulated as described in Sandri et al. (2004, A\&A, 428, 299) (hereafter Paper I). Given the overall constraints to the LFI optical design, we show that it is possible to improve the angular resolution by $5-7 \%$ by keeping the overall peak-to-peak signal of the Galaxy straylight contamination (GSC) below the level of few $\mu \mathrm{K}$ (and about 10 times smaller in terms of rms). A comparison between the level of straylight introduced by the different Galactic components for different beam regions (intermediate and far beam) is presented. We provide approximate relations, both for the intermediate and the far beam, for the rms and the peak-to-peak levels of the GSC as functions of the corresponding contributions to the integrated beam or of the spillover. For some reference cases we compare the results based on Galactic foreground maps derived from radio, IR, and $\mathrm{H} \alpha$ templates with those based on WMAP maps including CMB and extragalactic source fluctuations. The implications for the GSC in the PLANCK LFI polarization data are discussed. Finally, we compare the results obtained at $100 \mathrm{GHz}$ with those at $30 \mathrm{GHz}$, where the GSC is more critical.
\end{abstract}

Key words. cosmology: cosmic microwave background - Galaxy: general - space vehicles - telescopes - methods: data analysis

\section{Introduction}

The cosmic microwave background (CMB) anisotropies at few degree scales have been detected over the full sky by COBE/DMR (Smoot et al. 1992; Bennett et al. 1996;

* Appendices are only available in electronic form at http://www. edpsciences.org
Górski et al. 1996) and, in limited sky regions, by recent balloon-borne and ground experiments (see Bersanelli et al. 2002, and references therein for a review on the pre-WMAP observational status) characterized by high sensitivity and angular resolution, probing a universe model with $\Omega_{\text {tot }} \sim 1$ (see e.g. Netterfield et al. 2002; Stompor et al. 2001; 
Pryke et al. 2002, and references therein). The NASA WMAP (Wilkinson Microwave Anisotropy Probe, see Bennett et al. 2003a) space mission has made it possible to derive the angular power spectrum of the CMB anisotropy with unprecedent sensitivity and reliability (Hinshaw et al. 2003b; Kogut et al. 2003) and to improve the accuracy in the determination of the most important cosmological parameters (Spergel et al. 2003).

Future fundamental progress in the study of CMB anisotropy and polarization will be based on the PLANCK mission by ESA $^{1}$ (Bersanelli et al. 1996; Tauber 2001), planned to be launched in the year 2007.

In particular, the Low Frequency Instrument (LFI, Mandolesi et al. 1998; see also Mandolesi et al. 2002) and the High Frequency Instrument (HFI, Puget et al. 1998; see also Lamarre et al. 2002) on-board PLANCK will together cover a wide frequency range (30-900 GHz) which should significantly improve the accuracy of the subtraction of foreground contamination from the primordial CMB anisotropy, providing at the same time a gold mine of cosmological as well as astrophysical information (see e.g. De Zotti et al. 1999; Bouchet \& Gispert 1999, and references therein).

To reach these scientific goals, great attention has to be devoted to reducing and/or subtracting all the possible systematic effects.

The effect of optical distortions in the main beam (Burigana et al. 1998; Mandolesi et al. 2000; Page et al. 2003) and in the intermediate/far beam (Burigana et al. 2001; Barnes et al. 2003) has been widely recognized as the source of one of the most critical systematic errors for both balloon and space experiments.

The requirement for the rejection of unwanted radiation coming from directions far from the optical axis (straylight) is stringent for PLANCK and does not pertain only to the telescope itself, but to the entire optical system, including solar panels, shielding, thermal stability and focal assembly components. Variations of the spurious straylight signal introduce contaminations in the anisotropy measurements. The removal of this effect in data analysis is in principle much more complicated than the subtraction of the main beam distortion effect. This is due to the difficulty of knowing with high accuracy the "true" beam (by means of both ground and in-flight reconstruction techniques) at very low response levels.

The antenna response features far from the beam centre (spillover) are largely determined by rays coming from the feed that are reflected by the lower part of the subreflector and that are not intercepted by the main reflector, together with the rays coming directly from the feedhorn without any interaction with the reflecting structures; therefore, they can be reduced by decreasing the illumination at the edge of the primary (see Paper I). This will imply a lowering of the far beam level and the straylight contamination. On the other hand, this has a negative impact on the angular resolution for a given size of the primary mirror (see e.g. Mandolesi et al. 2000).

In the "cosmological window" between $\sim 70 \mathrm{GHz}$ and $\sim 300 \mathrm{GHz}$, where foreground contamination is minimum, it is extremely important to reach the best trade-off between the

\footnotetext{
${ }^{1}$ http://astro.estec.esa.nl/Planck/
}

improvement of the angular resolution, necessary to measure the high order acoustic peaks of CMB anisotropy, and the minimization of the straylight contamination due to the Galactic emission (GSC, Galaxy Straylight Contamination), one of the most critical systematic effects, most relevant at large and intermediate angular scales (i.e. at multipoles $\ell$ less than $\approx 100$ ).

In this work we focus, as a working case, on the $100 \mathrm{GHz}$ channels of the PLANCK Low Frequency Instrument, although the methods and the basic results described here can also be applied to other PLANCK frequency channels and to similar CMB anisotropy experiments. We shall also compare our results with simulated data at $30 \mathrm{GHz}$.

We will use here the results of the detailed computations concerning the full beam response for several optical configurations given in Paper I. We present extensive simulations of the GSC due to Galactic foreground components relevant at $100 \mathrm{GHz}$ in order to find the best compromise between resolution and GSC for the considered set of beams, as explained in the following.

The beam resolution mainly depends on the telescope and the considered frequency channel. On the other hand, a relative resolution improvement of $\sim 10 \%$, such as the one achievable by the kind of optimisation presented in these papers, is relevant for CMB anisotropy studies and in particular for the extraction of the $\mathrm{CMB}$ anisotropy angular power spectrum, $C_{\ell}$, at high multipoles, $\ell$, since, observationally, it is smoothed by the beam window function, $w_{\ell}: C_{\ell} \rightarrow \tilde{C}_{\ell}=C_{\ell} w_{\ell}$. For example, by improving the beam $F W H M\left(=\sqrt{8 \ln 2} \sigma_{\mathrm{b}}\right)$ from $11^{\prime}$ to $10^{\prime}$, the beam window function for a Gaussian symmetric beam, $w_{\ell} \simeq \exp \left[-\left(\ell \sigma_{\mathrm{b}}\right)^{2}\right]$, increases, respectively, by a factor 1.38 or 2.06 at $\ell=1000$ or 1500 . The same thing holds for the signal-to-noise ratio in the angular power spectrum determination for a given detector sensitivity.

The improvement in the resolution and in the corresponding signal to noise ratio in the $C_{\ell}$ determination at high multipoles (small angular scales) has to be achieved without introducing a significant degradation from straylight contamination in the quality of the signal and then in the final imaging of the CMB anisotropy map at large and intermediate angular scales (and, ultimately, in the $C_{\ell}$ determination at least at low and intermediate multipoles ${ }^{2}$ ). The nominal (i.e. related only to instrumental white noise) LFI sensitivity on pixels of $10^{\prime}$ $\left(30^{\prime}, 60^{\prime}\right)$ side $^{3}$ will be $\sim 12 \mu \mathrm{K}(\sim 4,2 \mu \mathrm{K})$ almost independently of the frequency channel. A given systematic error level implies a sensitivity degradation that could be "optimistically" added in quadrature to the white noise sensitivity, although a sum in modulo of the two errors is (albeit "conservative") certainly more realistic. Clearly a maximum acceptable GSC of $\sim 0.5 \mu \mathrm{K}$ (implying respectively on pixels of $10^{\prime}, 30^{\prime}, 60^{\prime}$ a sensitivity degradation of $\sim 4.2,12,25 \%$ if linearly added,

\footnotetext{
${ }^{2}$ At small angular scales (high multipoles), the evaluation of the GSC is complicated by the very long time required for full beam computations and, ultimately, by the difficulties in measuring the full beam with high precision and resolution. On the other hand, the GSC is expected to decrease with decreasing angular scale due to the relative decreasing variations of the beam at decreasing angular scales.

3 Approximately corresponding to $\approx \ell \sim 180 /(\theta / \mathrm{deg}) \sim 1000$, 400, 200.
} 
or of $\sim 0.087,0.78,3.1 \%$ if added in quadrature) represents a reasonable upper limit when referred to the whole set of data from (or to the whole sky obtained by) a given beam. Analogously, a maximum acceptable GSC or of $\sim 3 \mu \mathrm{K}$ (implying a sensitivity degradation of $\sim 25,75,150 \%$ if linearly added, or of $\sim 3,25,80 \%$ if added in quadrature) represents a reasonable upper limit when referred to a small fraction of data from (or a limited sky region obtained by) a given beam. Statistical estimators of the straylight signal such as the rms and the peak-to-peak value on the whole set of data can be adopted to characterise the GSC in the two cases considered above. We will search for optical configurations corresponding to the best resolutions that satisfy these GSC upper limit requirements.

In Sect. 2 we briefly describe the guidelines followed to simulate PLANCK observations (see also Appendix A in the electronic version of this paper or in Burigana \& Sáez 2003), the adopted optical input, and maps of Galactic components. The results of the simulations of straylight contamination are described in Sect. 3, while the comparison between the results obtained for different beams and foreground components is presented in Sect. 4 (and in Appendix B of the electronic version of this paper for a comparison with the straylight simulation at $30 \mathrm{GHz}$ ). Finally, we discuss the results and draw our main conclusions in Sect. 5.

\section{Simulations}

The selected orbit for PLANCK is a Lissajous orbit around the Lagrangian point L2 of the Sun-Earth system (see e.g. Mandolesi et al. 1998). The spacecraft spins at 1 r.p.m. and the field of view of the two instruments (LFI/HFI) is about $10^{\circ} \times 10^{\circ}$ centered at the telescope optical axis (the socalled telescope line of sight, LOS) at a given angle $\alpha$ from the spin-axis direction, given by a unit vector, $s$, chosen to be pointed in the opposite direction from the Sun. In this work we consider values of $\alpha \sim 85^{\circ}$, as adopted for the baseline scanning strategy. The spin axis will be kept parallel to the Sun-spacecraft direction and re-pointed by $\simeq 2.5^{\prime}$ once an hour (baseline scanning strategy). Hence PLANCK will trace large circles on the sky. A precession of the spin-axis with a period, $P$, of $\simeq 6$ months at a given angle $\beta \sim 10^{\circ}$ about an axis, $f$, parallel to the Sun-spacecraft direction (and away from the Sun) and shifted by $\simeq 2.5^{\prime}$ once an hour, may be included in the scanning strategy, possibly with a modulation of the speed of the precession in order to optimize data transmission (Bernard et al. 2002). Although the scanning strategy could be changed, the GSC pattern, the peak-to-peak signal, and the angular power spectrum are very weakly dependent on the details of these proposed scanning strategies (Burigana et al. 2000).

The code we have implemented to simulate PLANCK observations for a wide set of scanning strategies is described in detail in Burigana et al. (1997, 1998) and in Maino et al. (1999). In this study we exploit the baseline scanning strategy and simply assume that PLANCK is located in L2. We do not consider the PLANCK Lissajous orbit around L2 because its effects are negligible in this context.
We compute the convolutions between the beam response and the sky signal as described in Burigana et al. (2001) by taking resolutions of $\sim 1^{\circ}$ or $\sim 7^{\prime}$ for the far and the intermediate beam, respectively, and considering spin-axis shifts of $\sim 2^{\circ}$ every two days and 180 samplings per scan circle. In fact, the effects of beam features we want to study here occur on scales of a degree or larger and, also, the large set of optical simulations for the full beam is available at $\sim$ degree resolution (see Paper I for details on the computation time of optical simulations).

With respect to the reference frames described in Burigana et al. (2001), following the recent developments in optimizing the polarization properties of LFI main beams (see Paper I), the conversion between the standard Cartesian telescope frame $x, y, z$ and the beam frame $x_{\mathrm{bf}}, y_{\mathrm{bf}}, z_{\mathrm{bf}}$ requires a further angle $\psi_{\mathrm{B}}$ other than the standard polar coordinates $\theta_{\mathrm{B}}$ and $\phi_{\mathrm{B}}$ defining the colatitude and the longitude of the main beam centre direction in the telescope frame. Appendix A provides the transformation rules between the telescope frame and the beam frame, as well as the definition of the reference frames adopted in this work (see also Fig. 7 in Paper I).

The orientation of these frames as the satellite moves is implemented in the code. For each integration time, we determine the orientations in the sky of the telescope frame and of the beam frame, thus simultaneously performing a direct convolution between the full beam response and the sky signal for the desired number of maps.

\subsection{Optical inputs}

A detailed discussion of the optical simulation method and results is presented in Paper I (see Tables 2 and 3 of Paper I for the main properties of the adopted beams). We briefly summarize here the most relevant aspect in this context.

Several full beams have been simulated for different designs of two PLANCK LFI feedhorns at $100 \mathrm{GHz}$ located respectively in the most advantageous or disadvantageous position on the focal plane unit (see Paper I): three models (9A, 9B, 9C) for LFI9 and four models (4A, 4B, 4C, 4D) for LFI4. Different values of spillover will imply significant differences in the level of the GSC in the time ordered data (TOD).

The details of the beam response, computed as described in Paper I as functions of the two standard polar coordinates $\theta_{\mathrm{bf}}$, $\phi_{\mathrm{bf}}$ in the beam frame, also depend on the optical contributions considered in the analysis. For some representative cases, we will compare the results of our simulations of GSC adopting optical computations taking into account the first and second or the first, second and third order optical interactions (see Paper I).

In the beam frame we identify two different angular regions relevant for the straylight analysis: the intermediate beam and the far beam, respectively defined as the region between $1.2^{\circ}$ and $5^{\circ}$ from the beam centre direction and at angles larger than $5^{\circ}$ from the beam centre direction. The definition of these "reference" angles is, of course, somewhat arbitrary. They roughly separate angular regions where significant beam variations occur on sub-degree scales from those where they occur 

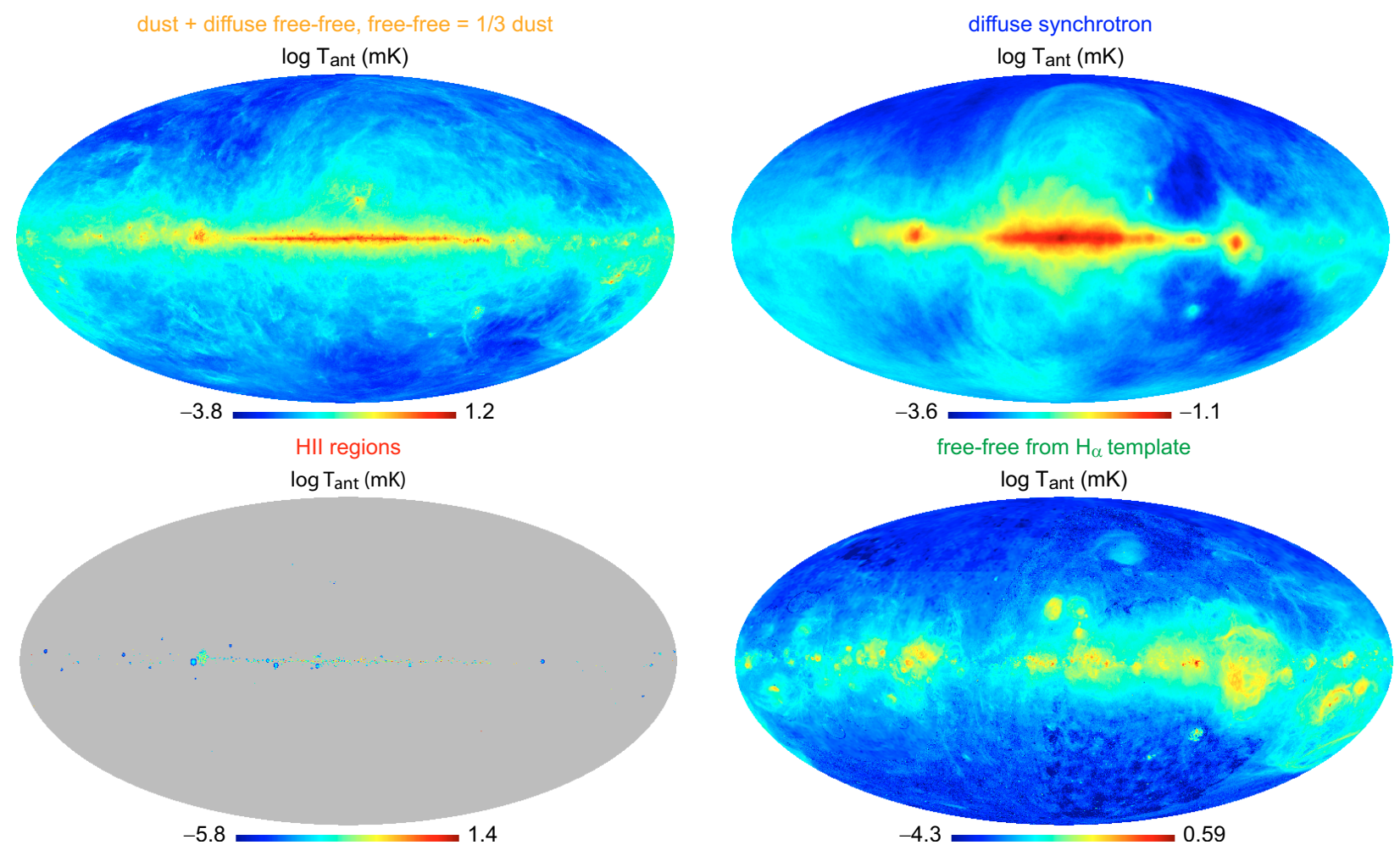

Fig. 1. Input maps at $100 \mathrm{GHz}$ adopted in this study in terms of antenna temperature on a logarithmic scale and in Galactic coordinates. Top left panel: map of the Galactic dust emission plus diffuse free-free emission, assumed to be correlated; top right panel: map of the Galactic diffuse synchrotron emission; bottom left panel: map of the free-free emission from compact Galactic HII regions; bottom right panel: map of the diffuse free-free emission derived from the $\mathrm{H} \alpha$ template (see also the text).

on scales of few degrees or larger. The values adopted here allow a direct comparison with a previous analysis (Burigana et al. 2001).

\subsection{Maps of galactic components}

In the cosmological window $(70-300 \mathrm{GHz})$ the CMB is clearly the dominant component; on the other hand the Galactic emission is still relevant at low and intermediate Galactic latitudes. The templates adopted here are similar to those described in Maino et al. (2002) and Paladini et al. (2003).

At $100 \mathrm{GHz}$ the most relevant Galactic component is the thermal dust emission. We adopted here a template obtained by extrapolating the maps by Schlegel et al. (1998) which combines IRAS and DIRBE data, assuming a grey-body spectrum (expressed in antenna temperature),

$T_{\mathrm{A}, \mathrm{dust}}(v) \propto \frac{\tilde{v}^{\beta+1}}{\mathrm{e}^{\tilde{v}}-1}, \tilde{v}=\frac{h v}{k T_{\text {dust }}}$

with uniform temperature $T_{\text {dust }}=18 \mathrm{~K}$ and emissivity $\beta=2$.

To simulate the free-free contribution we assume, somewhat arbitrarily, that it is perfectly correlated with the dust itself, i.e. that it has the same spatial distribution. Its antenna temperature scales with frequency as $T_{\mathrm{A}, \mathrm{ff}} \propto v^{-\beta_{\mathrm{ff}}}$, with $\beta_{\mathrm{ff}}=-2.1$. The relative amplitude of dust and free-free emission is assumed to be a factor of 3 at $100 \mathrm{GHz}$ (De Zotti et al. 1999). Thus, we produce a single map of "thermal" emission from dust plus diffuse free-free emission (see Fig. 1) with a spectrum described by:

$$
\begin{aligned}
T_{\mathrm{A}, \text { thermal }}(v)= & {\left[\frac{1}{3}\left(\frac{v}{100 \mathrm{GHz}}\right)^{\beta_{\mathrm{ff}}}+\frac{T_{\mathrm{A}, \mathrm{dust}}(v)}{T_{\mathrm{A}, \mathrm{dust}}(100 \mathrm{GHz})}\right] } \\
& \times T_{\mathrm{A}, \text { dust }}(100 \mathrm{GHz}) .
\end{aligned}
$$

We expect that the assumption of perfect spatial correlation between the templates of dust and free-free emission will produce straylight contamination levels slightly larger than those based on templates with similar surface brightness levels but uncorrelated. For some representative cases, we consider for comparison a free-free emission template derived from the $\mathrm{H} \alpha$ map by Finkbeiner $(2003)^{4}$ re-scaled to LFI frequencies according to the "initial model" of Sect. 5 of Bennett et al. (2003b), i.e. with a first rescaling to $K$-band antenna temperature using the conversion factor $11.4 \mu \mathrm{K} \mathrm{R}^{-1}$ followed by an extrapolation to higher frequencies with a power law spectral index $\beta_{\mathrm{ff}}=-2.15$ (see Fig. 1). A different map of "thermal" emission can then be obtained by adding the thermal dust emission template described at the beginning of this subsection to this free-free emission template.

The synchrotron emission template is the $408 \mathrm{MHz}$ map of Haslam et al. (1982), available at a resolution of $0.85^{\circ}$, extrapolated to the considered frequencies (see Fig. 1) assuming a uniform spectral index $\beta_{\text {syn }}=-2.9$ in antenna temperature. No attempt is made here to add small scale fluctuations, since the effects of beam features on which this work is focused occur

\footnotetext{
${ }^{4}$ http://www.astro.princeton.edu/ dfink/halpha/data/ index.html
} 
at degree or larger scales; for the same reason, the fact that the original template includes the convolution with the $0.85^{\circ}$ beam is not a concern.

Localized free-free emission dominates the signal on wide areas in the Galactic plane at least over the frequency range from 30 to $100 \mathrm{GHz}$. The Synthetic Catalog at $2.7 \mathrm{GHz}$ produced by Paladini et al. (2003) provides rich information on compact Galactic HII regions that has been used in this work to generate a map of free-free emission from these sources (see Fig. 1). A spectral index $\alpha=-2.1$ for thermal bremsstrahlung emission in a thin plasma in terms of antenna temperature has been adopted to extrapolate the signal from $2.7 \mathrm{GHz}$ to the considered PLANCK frequency channels. With respect to the simulated map reported by Paladini et al. (2003), we have implemented a code which simulates the contribution of each source in the Synthetic Catalog to each map pixel but does not perform the convolution with the beam. The convolution with the intermediate and far beam is applied at a subsequent stage as described above.

All maps have been projected according to the HEALPix scheme $^{5}$ (Hierarchical Equal Area and IsoLatitude Pixelization of the Sphere) by Gòrski et al. (1999).

When this work was nearly completed, the one year data products ${ }^{6}$ from the WMAP satellite became available. In a few representative cases, we have repeated the straylight analysis by adopting the WMAP frequency maps at 33 and $94 \mathrm{GHz}$, including in the straylight evaluation also the minor contributions to straylight signal from CMB and extragalactic source fluctuations ${ }^{7}$. Note that WMAP maps are convolved with the corresponding beams and include the effect of main beam distortions and straylight contamination as well as the instrumental noise and other sistematic effects not subtracted in the data analysis; on the other hand, these effects add only negligible contributions both because they are significantly smaller than the signal (Hinshaw et al. 2003a), therefore introducing only second order straylight effects, and because the straylight contamination is mainly contributed to by signal variations on degree or larger angular scales where systematic effects relevant at smaller scales average out.

\section{Simulation results}

The main output of our simulation code consists of the TOD of the signals entering the intermediate and far beam.

In the TOD of each scan circle, two prominent maxima typically appear. These are related, for the intermediate beam, to the two crossings of the Galactic plane of the telescope field of view and to the crossings of the Galactic plane of the main spillover in the case of the far beam. As already recognized by Burigana et al. (2001), these maxima are only slightly shifted with respect to the maxima of the signal entering the main beam

\footnotetext{
${ }^{5}$ http://www.eso.org/science/healpix/

${ }^{6}$ http://lambda.gsfc.nasa.gov

7 Note that none of these WMAP maps includes the monopole term, while our Galactic component maps include the corresponding monopoles. The comparison between the average straylight signals will therefore be only indicative. Clearly, anisotropy experiments are not sensitive to the monopole, which is directly subtracted in the data.
}

in the case of the intermediate beam and shifted by about $90^{\circ}$ in the case of the far beam, as a direct consequence of the beam shape.

The typical signal level is determined by the sky signal and the fraction, $f_{\%}=100 \int_{\Omega} J \mathrm{~d} \Omega / \int_{4 \pi} J \mathrm{~d} \Omega$, of the integrated antenna response in the considered beam region, $\underline{\Omega}$, reported in Table 3 of Paper I. The ratio between the fraction of the integrated antenna response in the far beam and in the intermediate beam provides only a rough upper limit to the ratio of straylight peak-to-peak signal in these two beam regions. In fact, while an extended very bright Galactic region could quite easily fill the (relatively small) solid angle subtended by the intermediate beam, the whole (quite large) solid angle subtented by the main spillover and by the other relevant far beam features cannot be easily filled by signals all coming simultaneously from very bright Galactic regions. Similarly, the fractional difference between the integrated antenna response in the far beam depending on whether the third order optical interaction is included in the computations or not provides an upper limit (less than about $10 \%$ ) to the fractional underestimation of the GSC when the third order optical interaction is neglected. More accurate estimates require numerical simulations.

In Figs. 2 and 3 we report the TOD corresponding to the straylight signal from the far and intermediate beam respectively for the three Galactic components described in Sect. 2.2. We consider, as a reference case, the beam LFI9 9B computed including the first, second and third order optical interactions.

The TOD corresponding to the difference between the straylight signal obtained including or neglecting the third order optical interaction are reported in Figs. 4 and 5. Clearly, the differences in the rms and peak-to-peak values of the straylight signal are within $\sim 5 \%$, i.e. smaller than the above upper limit derived on the basis of simple optical considerations by about a factor two. Therefore, considering only the first and second order optical interactions does not introduce a relevant loss of information in the optimization analysis of the optical design ${ }^{8}$.

Comparing Fig. 1 with Figs. 2 and 3, note how the different angular distribution of the three considered Galactic components is reflected in the TOD pattern which appears more or less "diffuse" according to the component considered.

Similar results are found for all the considered beams, but with a peak-to-peak and rms straylight signal significantly dependent on the adopted beam shape. A summary of the results obtained for the whole set of optical configurations is reported in the next section.

\section{Comparison between different beams and foreground components}

The statistical moments of the straylight signal TOD and the peak-to-peak value are reported in Tables 1-7 for our whole set of optical configurations at $100 \mathrm{GHz}$ (in the tables, the data referring to the global straylight effect from intermediate beam

\footnotetext{
8 On the contrary, the best knowledge of the beam, including all realistic effects, from dust and molecular contamination on mirror surfaces to mirror roughness and temperature behaviour, should be taken into account in the final data analysis.
} 

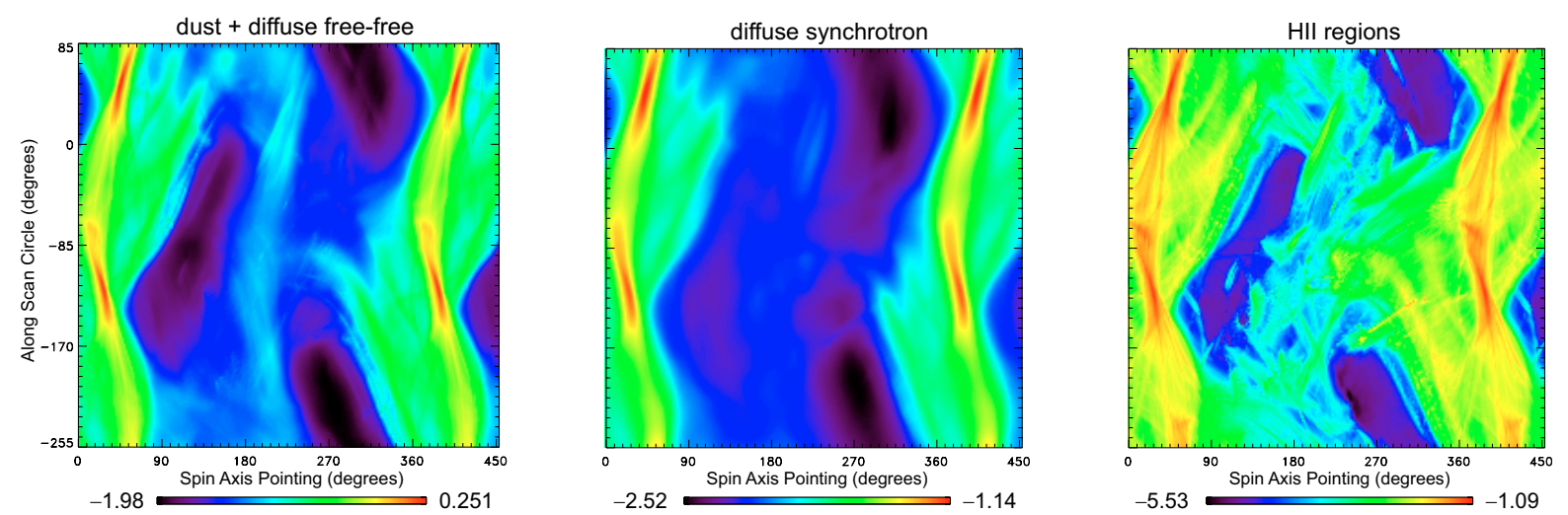

Fig. 2. TOD resulting from all scan circles for different Galactic components (left panel: dust emission plus diffuse free-free emission, assumed to be correlated; middle panel: diffuse synchrotron emission; right panel: free-free emission from compact Galactic HII regions). Units are the decimal logarithm of the antenna temperature in $\mu \mathrm{K}$. The ecliptic coordinates refer here to the latitude of the telescope LOS (for graphic purposes, in this plot the range between $-85^{\circ}$ and $-255^{\circ}$ refers to the second half of each scan circle) and to its longitude shift with respect to its initial direction, or equivalently to the shift of the spin axis pointing direction. We report here the straylight signal in the far beam computed including the first, second and third order optical interactions for the beam LFI9 9B (see also the text).
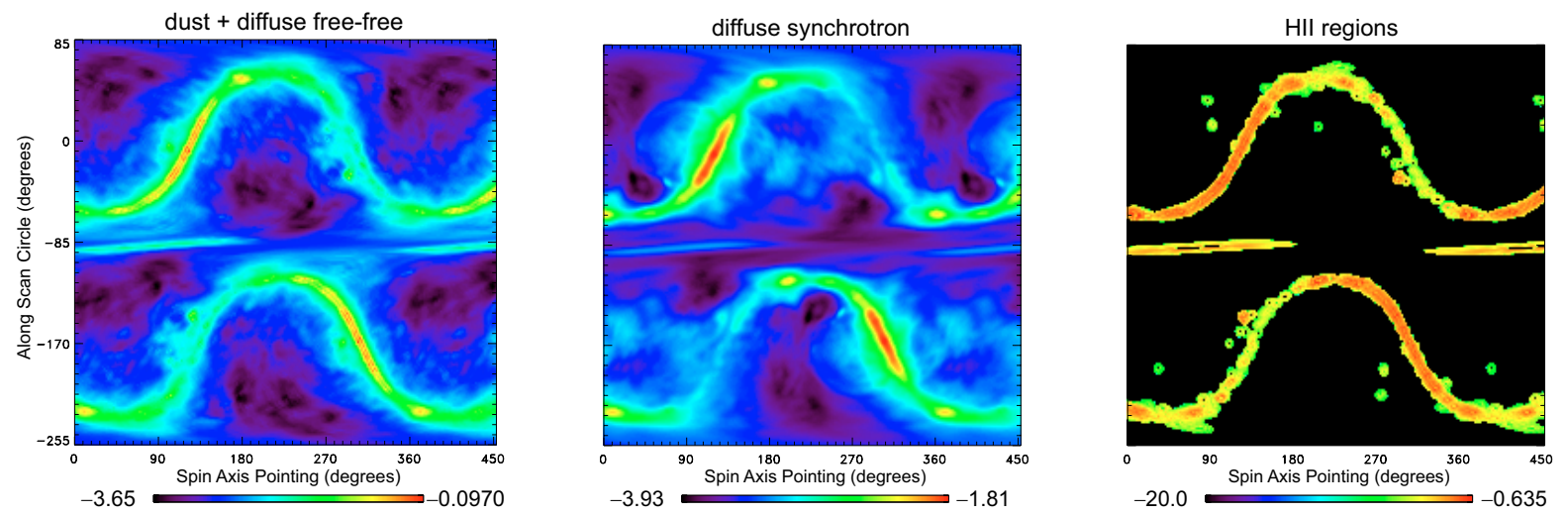

Fig. 3. The same as in Fig. 2, but for the signal from the intermediate beam. In this case, Galactic HII regions produce a quite localized straylight contamination which, in particular, is equal to zero far from these sources and arbitrarly set to $10^{-20} \mu \mathrm{K}$ for graphic purposes (as in Fig. 2, units are the decimal logarithm of the antenna temperature in $\mu \mathrm{K}$; see also the text).
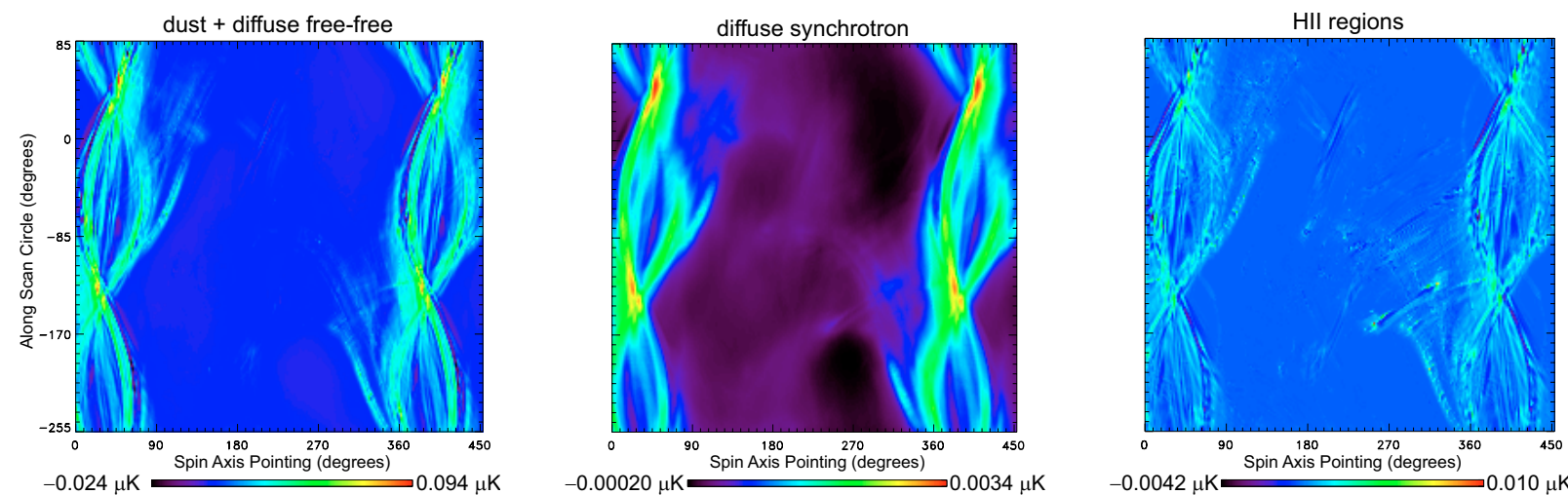

Fig. 4. The same as in Fig. 2, but referring to the difference between the straylight signals computed by including or not the third order optical interaction (the antenna temperature is reported here in $\mu \mathrm{K}$ on a linear scale; see also the text).

plus far beam $(\mathrm{I}+\mathrm{F})$ are derived including also the contribution from the third order optical interactions, when available). Table 8 summarizes the basic information contained in the above tables as a function of the spillover.

It appears from the tables that the contamination from the far beam is much more relevant than that from the intermediate beam when the rms of the TOD is considered; looking at the peak-to-peak signal of the straylight, the contamination from the far beam is larger by a factor of a few or comparable to that from the intermediate beam. The larger impact of the far beam than that of the intermediate beam is particularly remarkable for the case of the diffuse Galactic components, while it is less evident in the case of the Galactic HII region map. We find in fact a quite general behaviour: the more diffuse the 

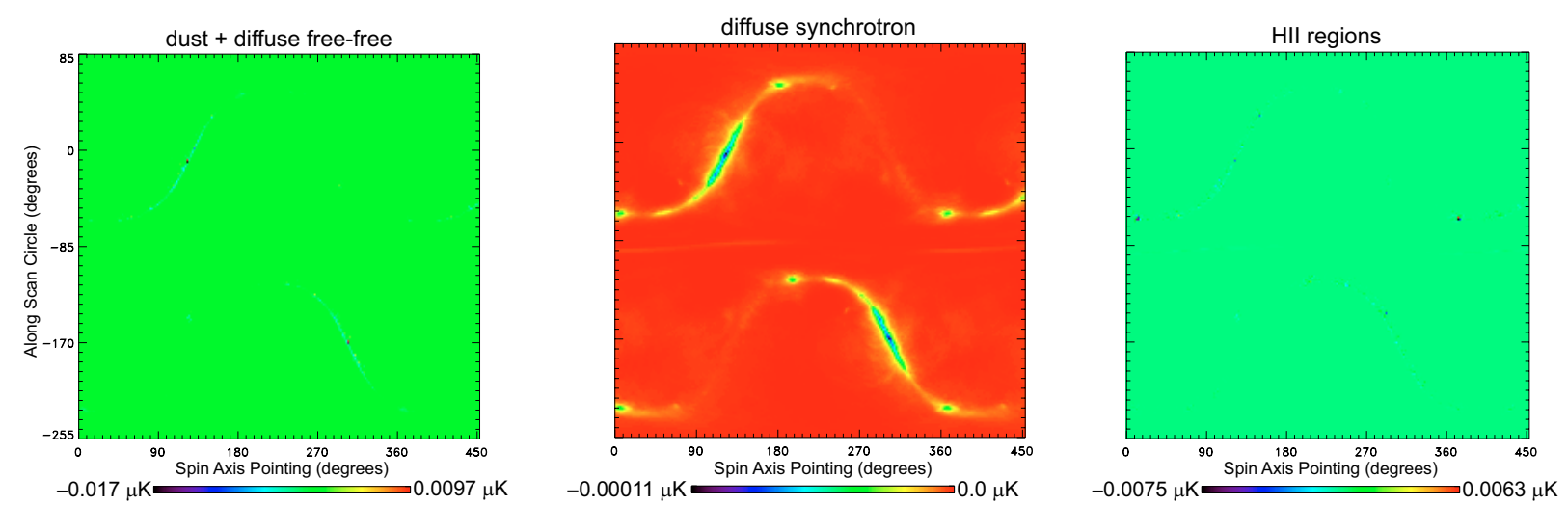

Fig. 5. The same as in Fig. 4, but for the signal from the intermediate beam (see also the text).

Table 1. Statistical moments and peak-to-peak difference of the simulated TOD (antenna temperature expressed in $\mu \mathrm{K}$ ) from the intermediate and far beam of the simulated beam LFI9 9A at $100 \mathrm{GHz}$ for different Galactic components. The row labelled with "I12" refers to the signal in the intermediate beam computed taking into account the first and second order optical interactions. The row labelled with "F123" ("F12") refers to the signal in the far beam computed taking into account the first, second and third (first and second) order optical interactions (see also the text).

\begin{tabular}{|c|c|c|c|c|c|c|}
\hline \multicolumn{7}{|c|}{ LFI9 9A } \\
\hline Beam & & & & & Skewness & Kurtosis \\
\hline Region & Average & Variance & $\mathrm{rms}$ & Peak-to-peak & Index & Index \\
\hline \multicolumn{7}{|c|}{ Dust + "correlated" diffuse free-free emission } \\
\hline $\mathrm{I} 12$ & $2.27 \times 10^{-3}$ & $5.81 \times 10^{-5}$ & $7.63 \times 10^{-3}$ & $2.61 \times 10^{-1}$ & $9.45 \times 10^{+0}$ & $1.33 \times 10^{+2}$ \\
\hline F123 & $6.71 \times 10^{-2}$ & $4.77 \times 10^{-3}$ & $6.90 \times 10^{-2}$ & $5.88 \times 10^{-1}$ & $1.88 \times 10^{+0}$ & $4.92 \times 10^{+0}$ \\
\hline F12 & $6.40 \times 10^{-2}$ & $4.49 \times 10^{-3}$ & $6.70 \times 10^{-2}$ & $5.68 \times 10^{-1}$ & $1.89 \times 10^{+0}$ & $4.90 \times 10^{+0}$ \\
\hline $\mathrm{I}+\mathrm{F}$ & $6.93 \times 10^{-2}$ & $4.83 \times 10^{-3}$ & $6.95 \times 10^{-2}$ & $5.88 \times 10^{-1}$ & $1.81 \times 10^{+0}$ & $4.59 \times 10^{+0}$ \\
\hline \multicolumn{7}{|c|}{ Diffuse synchrotron emission } \\
\hline $\mathrm{I} 12$ & $2.10 \times 10^{-4}$ & $1.05 \times 10^{-7}$ & $3.24 \times 10^{-4}$ & $4.66 \times 10^{-3}$ & $6.59 \times 10^{+0}$ & $5.64 \times 10^{+1}$ \\
\hline F123 & $5.87 \times 10^{-3}$ & $1.65 \times 10^{-5}$ & $4.07 \times 10^{-3}$ & $2.32 \times 10^{-2}$ & $1.27 \times 10^{+0}$ & $1.11 \times 10^{+0}$ \\
\hline $\mathrm{F} 12$ & $5.60 \times 10^{-3}$ & $1.55 \times 10^{-5}$ & $3.94 \times 10^{-3}$ & $2.24 \times 10^{-2}$ & $1.29 \times 10^{+0}$ & $1.13 \times 10^{+0}$ \\
\hline $\mathrm{I}+\mathrm{F}$ & $6.08 \times 10^{-3}$ & $1.67 \times 10^{-5}$ & $4.09 \times 10^{-3}$ & $2.31 \times 10^{-2}$ & $1.22 \times 10^{+0}$ & $9.44 \times 10^{-1}$ \\
\hline \multicolumn{7}{|c|}{ H II regions } \\
\hline $\mathrm{I} 12$ & $7.70 \times 10^{-5}$ & $7.45 \times 10^{-7}$ & $8.63 \times 10^{-4}$ & $8.05 \times 10^{-2}$ & $4.14 \times 10^{+1}$ & $2.67 \times 10^{+3}$ \\
\hline F123 & $2.13 \times 10^{-3}$ & $7.14 \times 10^{-6}$ & $2.67 \times 10^{-3}$ & $2.67 \times 10^{-2}$ & $1.93 \times 10^{+0}$ & $5.87 \times 10^{+0}$ \\
\hline F12 & $2.02 \times 10^{-3}$ & $6.69 \times 10^{-6}$ & $2.59 \times 10^{-3}$ & $2.61 \times 10^{-2}$ & $1.94 \times 10^{+0}$ & $5.82 \times 10^{+0}$ \\
\hline $\mathrm{I}+\mathrm{F}$ & $2.20 \times 10^{-3}$ & $7.99 \times 10^{-6}$ & $2.83 \times 10^{-3}$ & $8.67 \times 10^{-2}$ & $2.97 \times 10^{+0}$ & $3.27 \times 10^{+1}$ \\
\hline \multicolumn{7}{|c|}{ Sum of the above components } \\
\hline $\mathrm{I} 12$ & $2.55 \times 10^{-3}$ & $7.02 \times 10^{-5}$ & $8.38 \times 10^{-3}$ & $2.94 \times 10^{-1}$ & $9.51 \times 10^{+0}$ & $1.37 \times 10^{+2}$ \\
\hline F123 & $7.51 \times 10^{-2}$ & $5.71 \times 10^{-3}$ & $7.56 \times 10^{-2}$ & $6.38 \times 10^{-1}$ & $1.84 \times 10^{+0}$ & $4.66 \times 10^{+0}$ \\
\hline $\mathrm{F} 12$ & $7.16 \times 10^{-2}$ & $5.38 \times 10^{-3}$ & $7.33 \times 10^{-2}$ & $6.16 \times 10^{-1}$ & $1.85 \times 10^{+0}$ & $4.64 \times 10^{+0}$ \\
\hline $\mathrm{I}+\mathrm{F}$ & $7.76 \times 10^{-2}$ & $5.79 \times 10^{-3}$ & $7.61 \times 10^{-2}$ & $6.38 \times 10^{-1}$ & $1.78 \times 10^{+0}$ & $4.34 \times 10^{+0}$ \\
\hline
\end{tabular}

component, the more relevant the straylight contamination from the far beam with respect to the intermediate beam.

On the contrary, the skewness and kurtosis indices are larger for the straylight contamination from the intermediate beam because they are more sensitive to localized features. As expected, these indices are larger for the free-free emission from Galactic HII regions than for the more diffuse components.

Overall, the GSC from the intermediate beam, relevant at relatively low Galactic latitudes, has less impact on the extraction of the CMB science from the data than that from the far beam which is important also at intermediate and high latitudes $^{9}$.

For the representative case of LFI9 9B we evaluated the straylight signal on the basis of the WMAP map at $94 \mathrm{GHz}$ both for the intermediate and far beam (see Table 2); for the straylight from the far beam we also considered this input map for the case of LFI4 4A (see Table 4). For the signal in the intermediate beam, we find a straylight contamination larger

\footnotetext{
${ }^{9}$ In fact, albeit some attempts to avoid Galactic cuts in CMB anisotropy data analysis are being made, regions at low Galactic latitudes are typically removed for the analysis of the CMB.
} 
Table 2. The same as in Table 1, but for the indicated simulated beam. The row labelled with "I123" refers to the signal in the intermediate beam computed taking into account the first, second and third order optical interactions. Note how the straylight in the intermediate beam is slightly smaller when the third order optical interaction is included (compare I123 with I12). This is due to a small decrease ( $\simeq 0.4 \%)$ of the integrated beam response in that beam region when the third order optical interaction is taken into account because of the combination in amplitude and phase of the various contributions, producing an overall antenna response - different from a simple sum of the powers of the various contributions - that typically, but not necessarily, increases with the number of considered contributions, as occurs here in the far beam. Also reported is the straylight contamination from this free-free emission as derived from the $\mathrm{H} \alpha$ map and the combined straylight from such free-free emission template and dust emission without assuming a correlation between dust and free-free emission. In this case, we quote for comparison the results for the straylight from the intermediate beam computed with a physical optics calculation without including the shields (IPOWoS). Finally, we summarize the results obtained with the WMAP map at $94 \mathrm{GHz}$, including all components, and, for the far beam, those obtained by keeping only the region at $|b| \leq 10^{\circ}$ (see also the text).

\begin{tabular}{|c|c|c|c|c|c|c|}
\hline \multicolumn{7}{|c|}{ LFI9 9B } \\
\hline Beam & & & & & Skewness & Kurtosis \\
\hline Region & Average & Variance & rms & Peak-to-peak & Index & Index \\
\hline \multicolumn{7}{|c|}{ Dust + "correlated" diffuse free-free emission } \\
\hline IPOWoS & $7.66 \times 10^{-3}$ & $6.53 \times 10^{-4}$ & $2.55 \times 10^{-2}$ & $7.94 \times 10^{-1}$ & $9.14 \times 10^{+0}$ & $1.20 \times 10^{+2}$ \\
\hline $\mathrm{I} 123$ & $7.54 \times 10^{-3}$ & $6.38 \times 10^{-4}$ & $2.53 \times 10^{-2}$ & $8.00 \times 10^{-1}$ & $9.24 \times 10^{+0}$ & $1.23 \times 10^{+2}$ \\
\hline $\mathrm{I} 12$ & $7.57 \times 10^{-3}$ & $6.41 \times 10^{-4}$ & $2.53 \times 10^{-2}$ & $7.90 \times 10^{-1}$ & $9.18 \times 10^{+0}$ & $1.21 \times 10^{+2}$ \\
\hline F123 & $1.45 \times 10^{-1}$ & $3.31 \times 10^{-2}$ & $1.82 \times 10^{-1}$ & $1.77 \times 10^{+0}$ & $2.88 \times 10^{+0}$ & $1.19 \times 10^{+1}$ \\
\hline F12 & $1.35 \times 10^{-1}$ & $3.03 \times 10^{-2}$ & $1.74 \times 10^{-1}$ & $1.73 \times 10^{+0}$ & $2.96 \times 10^{+0}$ & $1.26 \times 10^{+1}$ \\
\hline $\mathrm{I}+\mathrm{F}$ & $1.53 \times 10^{-1}$ & $3.35 \times 10^{-2}$ & $1.83 \times 10^{-1}$ & $1.77 \times 10^{+0}$ & $2.77 \times 10^{+0}$ & $1.12 \times 10^{+1}$ \\
\hline \multicolumn{7}{|c|}{ Diffuse synchrotron emission } \\
\hline IPOWoS & $7.08 \times 10^{-4}$ & $1.19 \times 10^{-6}$ & $1.09 \times 10^{-3}$ & $1.57 \times 10^{-2}$ & $6.57 \times 10^{+0}$ & $5.60 \times 10^{+1}$ \\
\hline $\mathrm{I} 123$ & $6.96 \times 10^{-4}$ & $1.16 \times 10^{-6}$ & $1.08 \times 10^{-3}$ & $1.55 \times 10^{-2}$ & $6.58 \times 10^{+0}$ & $5.63 \times 10^{+1}$ \\
\hline $\mathrm{I} 12$ & $6.99 \times 10^{-4}$ & $1.17 \times 10^{-6}$ & $1.08 \times 10^{-3}$ & $1.55 \times 10^{-2}$ & $6.57 \times 10^{+0}$ & $5.61 \times 10^{+1}$ \\
\hline F123 & $1.27 \times 10^{-2}$ & $1.06 \times 10^{-4}$ & $1.03 \times 10^{-2}$ & $6.93 \times 10^{-2}$ & $1.89 \times 10^{+0}$ & $3.97 \times 10^{+0}$ \\
\hline F12 & $1.18 \times 10^{-2}$ & $9.63 \times 10^{-5}$ & $9.81 \times 10^{-3}$ & $6.71 \times 10^{-2}$ & $1.95 \times 10^{+0}$ & $4.24 \times 10^{+0}$ \\
\hline $\mathrm{I}+\mathrm{F}$ & $1.34 \times 10^{-2}$ & $1.07 \times 10^{-4}$ & $1.03 \times 10^{-2}$ & $6.91 \times 10^{-2}$ & $1.81 \times 10^{+0}$ & $3.67 \times 10^{+0}$ \\
\hline \multicolumn{7}{|c|}{ H II regions } \\
\hline IPOWoS & $2.62 \times 10^{-4}$ & $7.39 \times 10^{-6}$ & $2.72 \times 10^{-3}$ & $2.34 \times 10^{-1}$ & $3.55 \times 10^{+1}$ & $2.03 \times 10^{+3}$ \\
\hline I123 & $2.58 \times 10^{-4}$ & $7.31 \times 10^{-6}$ & $2.70 \times 10^{-3}$ & $2.32 \times 10^{-1}$ & $3.57 \times 10^{+1}$ & $2.03 \times 10^{+3}$ \\
\hline $\mathrm{I} 12$ & $2.59 \times 10^{-4}$ & $7.31 \times 10^{-6}$ & $2.70 \times 10^{-3}$ & $2.32 \times 10^{-1}$ & $3.55 \times 10^{+1}$ & $2.02 \times 10^{+3}$ \\
\hline F123 & $4.45 \times 10^{-3}$ & $4.70 \times 10^{-5}$ & $6.86 \times 10^{-3}$ & $8.09 \times 10^{-2}$ & $3.18 \times 10^{+0}$ & $1.60 \times 10^{+1}$ \\
\hline $\mathrm{F} 12$ & $4.13 \times 10^{-3}$ & $4.27 \times 10^{-5}$ & $6.54 \times 10^{-3}$ & $7.64 \times 10^{-2}$ & $3.27 \times 10^{+0}$ & $1.68 \times 10^{+1}$ \\
\hline $\mathrm{I}+\mathrm{F}$ & $4.71 \times 10^{-3}$ & $5.45 \times 10^{-5}$ & $7.38 \times 10^{-3}$ & $2.45 \times 10^{-1}$ & $4.38 \times 10^{+0}$ & $5.24 \times 10^{+1}$ \\
\hline
\end{tabular}

by a factor $\simeq 1.6$ in terms of rms (but essentially unchanged in terms of peak-to-peak value). It is interesting to note that, since the $94 \mathrm{GHz}$ WMAP channel has a low level of Galactic foreground contamination, the contribution to straylight from CMB fluctuations clearly appears far from the Galactic plane (see Fig. 6, right image) when this map is used ${ }^{10}$. In fact, simulations conducted by means of the three Galactic templates show that the straylight signal is negligible at intermediate and high latitudes (see Fig. 6, left image). If we set to zero the values of the TOD samples with signal smaller than $\simeq 0.15 \mu \mathrm{K}$ (the maximum value of straylight far from the Galactic plane in the right panel of Fig. 6) we obtain a rms straylight of $\simeq 0.024 \mu \mathrm{K}$, quite close to the rms straylight value of $\simeq 0.028 \mu \mathrm{K}$ obtained in the case of the sum of the three adopted Galactic templates

\footnotetext{
${ }^{10}$ On the contrary, this effect is not evident at $30 \mathrm{GHz}$ (see Fig. B.1 in Appendix B).
}

(see Table 2). This means that the main contribution to the factor $\simeq 1.6$ discrepancy in the rms of the straylight signal found adopting either the three Galactic templates or the WMAP map at $94 \mathrm{GHz}$ is mainly produced by the CMB anisotropy. For the configuration 9B of LFI9 we have repeated the analysis for the intermediate beam evaluated with a physical optics computation but neglecting the shields ${ }^{11}$ (see Paper I). As evident from Table 2, the GSC results found in this case are typically just different from (and typically worse than) those based on the multi-reflector geometrical theory of diffraction including the shields, although this difference is so small as to be negligible in practice.

The straylight contamination in the far beam is larger by a factor $\sim 1.3$, in terms of both rms and peak-to-peak value,

\footnotetext{
11 Of course, including the shields is necessary for the far beam computation. A physical optics computation is not feasible in this case.
} 
Table 2. continued.

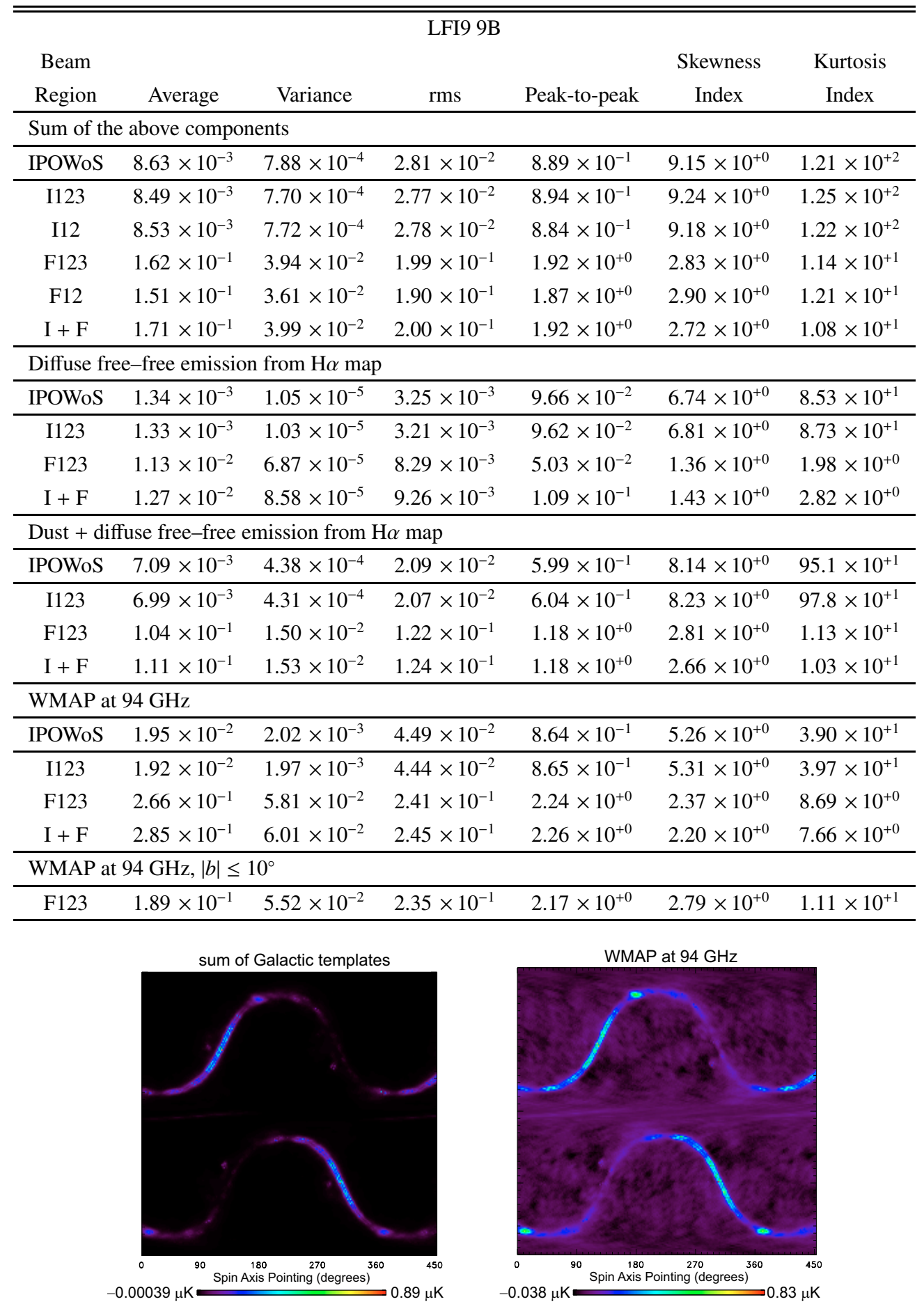

Fig. 6. The same as in Fig. 3 but for the sum of the three Galactic templates and for the WMAP map at 94 GHz where the contribution from $\mathrm{CMB}$ anisotropy appears (the antenna temperature is reported hwere in $\mu \mathrm{K}$ on a linear scale; see also the text).

when the WMAP map is used rather than the sum of the three adopted Galactic templates. The above CMB anisotropy effect is not evident in this case, in agreement with the idea that the contribution from CMB anisotropies (and also that from extragalactic bright sources and fluctuations, minimal at about $100-200 \mathrm{GHz}$, and from the instrumental noise) contained in the WMAP map, can produce only minor contaminations, compared to the Galactic signal, because of their smaller power at large angular scales which implies that positive and negative fluctuations partially compensate inside the relatively large beam solid angles relevant for this kind of straylight contamination. In addition, the regions at low straylight signal in the simulation based on the WMAP map show a pattern that, although with different signal values, is quite similar to that 
Table 3. The same as in Table 1, but for the indicated simulated beam.

\begin{tabular}{|c|c|c|c|c|c|c|}
\hline \multicolumn{7}{|c|}{ LFI9 9C } \\
\hline Beam & & & & & Skewness & Kurtosis \\
\hline Region & Average & Variance & rms & Peak-to-peak & Index & Index \\
\hline \multicolumn{7}{|c|}{ Dust + "correlated" diffuse free-free emission } \\
\hline $\mathrm{I} 12$ & $2.20 \times 10^{-2}$ & $5.42 \times 10^{-3}$ & $7.36 \times 10^{-2}$ & $2.02 \times 10^{+0}$ & $8.87 \times 10^{+0}$ & $1.06 \times 10^{+2}$ \\
\hline F123 & $4.71 \times 10^{-1}$ & $4.17 \times 10^{-1}$ & $6.46 \times 10^{-1}$ & $5.84 \times 10^{+0}$ & $3.00 \times 10^{+0}$ & $1.24 \times 10^{+1}$ \\
\hline $\mathrm{F} 12$ & $4.34 \times 10^{-1}$ & $3.80 \times 10^{-1}$ & $6.16 \times 10^{-1}$ & $5.70 \times 10^{+0}$ & $3.10 \times 10^{+0}$ & $1.32 \times 10^{+1}$ \\
\hline $\mathrm{I}+\mathrm{F}$ & $4.93 \times 10^{-1}$ & $4.20 \times 10^{-1}$ & $6.48 \times 10^{-1}$ & $5.84 \times 10^{+0}$ & $2.92 \times 10^{+0}$ & $1.19 \times 10^{+1}$ \\
\hline \multicolumn{7}{|c|}{ Diffuse synchrotron emission } \\
\hline $\mathrm{I} 12$ & $2.04 \times 10^{-3}$ & $9.95 \times 10^{-6}$ & $3.15 \times 10^{-3}$ & $4.59 \times 10^{-2}$ & $6.65 \times 10^{+0}$ & $5.76 \times 10^{+1}$ \\
\hline F123 & $4.12 \times 10^{-2}$ & $1.33 \times 10^{-3}$ & $3.65 \times 10^{-2}$ & $2.45 \times 10^{-1}$ & $2.04 \times 10^{+0}$ & $4.61 \times 10^{+0}$ \\
\hline F12 & $3.79 \times 10^{-2}$ & $1.19 \times 10^{-3}$ & $3.45 \times 10^{-2}$ & $2.38 \times 10^{-1}$ & $2.12 \times 10^{+0}$ & $5.03 \times 10^{+0}$ \\
\hline $\mathrm{I}+\mathrm{F}$ & $4.32 \times 10^{-2}$ & $1.33 \times 10^{-3}$ & $3.65 \times 10^{-2}$ & $2.44 \times 10^{-1}$ & $1.98 \times 10^{+0}$ & $4.39 \times 10^{+0}$ \\
\hline \multicolumn{7}{|c|}{ H II regions } \\
\hline $\mathrm{I} 12$ & $7.54 \times 10^{-4}$ & $5.36 \times 10^{-5}$ & $7.32 \times 10^{-3}$ & $5.46 \times 10^{-1}$ & $2.98 \times 10^{+1}$ & $1.41 \times 10^{+3}$ \\
\hline F123 & $1.43 \times 10^{-2}$ & $5.92 \times 10^{-4}$ & $2.43 \times 10^{-2}$ & $2.75 \times 10^{-1}$ & $3.29 \times 10^{+0}$ & $1.58 \times 10^{+1}$ \\
\hline F12 & $1.31 \times 10^{-2}$ & $5.37 \times 10^{-4}$ & $2.32 \times 10^{-2}$ & $2.59 \times 10^{-1}$ & $3.39 \times 10^{+0}$ & $1.68 \times 10^{+1}$ \\
\hline $\mathrm{I}+\mathrm{F}$ & $1.50 \times 10^{-2}$ & $6.47 \times 10^{-4}$ & $2.54 \times 10^{-2}$ & $5.91 \times 10^{-1}$ & $3.62 \times 10^{+0}$ & $2.42 \times 10^{+1}$ \\
\hline \multicolumn{7}{|c|}{ Sum of the above components } \\
\hline $\mathrm{I} 12$ & $2.48 \times 10^{-2}$ & $6.52 \times 10^{-3}$ & $8.08 \times 10^{-2}$ & $2.26 \times 10^{+0}$ & $8.85 \times 10^{+0}$ & $1.07 \times 10^{+2}$ \\
\hline F123 & $5.26 \times 10^{-1}$ & $4.97 \times 10^{-1}$ & $7.05 \times 10^{-1}$ & $6.35 \times 10^{+0}$ & $2.96 \times 10^{+0}$ & $1.20 \times 10^{+1}$ \\
\hline $\mathrm{F} 12$ & $4.85 \times 10^{-1}$ & $4.52 \times 10^{-1}$ & $6.72 \times 10^{-1}$ & $6.18 \times 10^{+0}$ & $3.05 \times 10^{+0}$ & $1.27 \times 10^{+1}$ \\
\hline $\mathrm{I}+\mathrm{F}$ & $5.51 \times 10^{-1}$ & $5.00 \times 10^{-1}$ & $7.07 \times 10^{-1}$ & $6.35 \times 10^{+0}$ & $2.88 \times 10^{+0}$ & $1.15 \times 10^{+1}$ \\
\hline
\end{tabular}

found in the same regions using the Galactic templates. These considerations support the idea that the difference by a factor $\sim 1.3$ is mainly due to differences in the Galactic components. The straylight contamination from Galactic synchrotron emission and HII regions is relatively weak at $100 \mathrm{GHz}$ compared to that from free-free and dust emission. For the representative case of LFI9 9B, the simulations carried out using the dust template added to the free-free map extrapolated from the $\mathrm{H} \alpha$ template show a GSC level smaller less than that obtained assuming an exact correlation between free-free and dust emission; in addition, the GSC obtained considering only the free-free map extrapolated from the $\mathrm{H} \alpha$ template is $\sim 6$ times smaller (in terms of both rms and peak-to-peak value) than that obtained from the correlated free-free and dust emission template (see Table 2). Finally, a simulation carried out using the WMAP map and considering only the regions at Galactic latitude $|b| \leq 10^{\circ}$ (see last row of Table 2) gives results close to those obtained without cuts. We then conclude that the differences found using the WMAP map or the adopted templates are mainly due to differences in the dust emission component.

Exploiting our set of optical configurations (and taking into account the correction factor, $\simeq 1.3$, found on the basis of the WMAP map for the straylight from the far beam), we find a linear approximation describing quite well the dependence of the rms and the peak-to-peak values of the GSC at $100 \mathrm{GHz}$ on the fractional contribution, $f_{\%}$, to the integrated beam response from the considered beam region (see also Fig. 7):

peak-to-peak $\simeq 5.3 \mu \mathrm{K} \times f_{\%} \simeq(1.8-3.0) \mu \mathrm{K} \times$ spillover

$\mathrm{rms} \simeq 0.62 \mu \mathrm{K} \times f_{\%} \simeq(0.21-0.35) \mu \mathrm{K} \times$ spillover for the far beam, and

peak-to-peak $\simeq 28 \mu \mathrm{K} \times f_{\%} \simeq 1.3 \mu \mathrm{K} \times$ spillover

$\mathrm{rms} \simeq 0.86 \mu \mathrm{K} \times f_{\%} \simeq 0.041 \mu \mathrm{K} \times$ spillover

for the intermediate beam ${ }^{12}$, where Eqs. (1)-(5) of Paper I are taken into account in the last equalities of these relations. The numerical coefficients in Eq. (6) can be multiplied by $\simeq 1.6$ to include also the straylight contribution in the intermediate beam from CMB fluctuations. Note the spread (within a factor $\simeq 2$ ) of the proportionality coefficient between the value of $f_{\%}$ of the far beam and the spillover for the considered optical configurations (while for the intermediate beam it changes very little; see Eqs. (1)-(5) of Paper I). It implies a similar spread in the relationship between the straylight contamination and the spillover. Figure 7 shows that the above relations are accurate within $\sim \pm 20 \%$.

Clearly, in the cosmological window the GSC contamination is less than that it is in the lowest and highest PLANCK frequency channels and so its impact on CMB power spectrum recovery (see Burigana et al. 2001 for an analysis of GSC impact on the recovery of the power spectrum and on the Fourier decomposition of scan circle data in the lowest PLANCK frequency channel). On the other hand, the ultimate goal of PLANCK, and in general of future CMB anisotropy experiments after WMAP, is not only the power spectrum

\footnotetext{
${ }^{12}$ In these fits we adopt, for uniformity, the numbers found considering the first and second order optical interactions, available for all cases.
} 
Table 4. The same as in Table 1, but for the indicated simulated beam. For the straylight in the far beam we also report the result based on the WMAP map at $94 \mathrm{GHz}$, including all components.

\begin{tabular}{|c|c|c|c|c|c|c|}
\hline \multicolumn{7}{|c|}{ LFI4 4A } \\
\hline Beam & & & & & Skewness & Kurtosis \\
\hline Region & Average & Variance & rms & Peak-to-peak & Index & Index \\
\hline \multicolumn{7}{|c|}{ Dust + "correlated" diffuse free-free emission } \\
\hline $\mathrm{I} 12$ & $5.03 \times 10^{-4}$ & $2.88 \times 10^{-6}$ & $1.70 \times 10^{-3}$ & $5.71 \times 10^{-2}$ & $9.19 \times 10^{+0}$ & $1.22 \times 10^{+2}$ \\
\hline F123 & $1.42 \times 10^{-2}$ & $1.83 \times 10^{-4}$ & $1.35 \times 10^{-2}$ & $1.02 \times 10^{-1}$ & $1.73 \times 10^{+0}$ & $4.00 \times 10^{+0}$ \\
\hline F12 & $1.24 \times 10^{-2}$ & $1.54 \times 10^{-4}$ & $1.24 \times 10^{-2}$ & $1.02 \times 10^{-1}$ & $2.02 \times 10^{+0}$ & $5.84 \times 10^{+0}$ \\
\hline $\mathrm{I}+\mathrm{F}$ & $1.47 \times 10^{-2}$ & $1.87 \times 10^{-4}$ & $1.37 \times 10^{-2}$ & $1.02 \times 10^{-1}$ & $1.65 \times 10^{+0}$ & $3.58 \times 10^{+0}$ \\
\hline \multicolumn{7}{|c|}{ Diffuse synchrotron emission } \\
\hline I12 & $4.62 \times 10^{-5}$ & $5.13 \times 10^{-9}$ & $7.16 \times 10^{-5}$ & $1.07 \times 10^{-3}$ & $6.72 \times 10^{+0}$ & $5.96 \times 10^{+1}$ \\
\hline F123 & $1.24 \times 10^{-3}$ & $5.89 \times 10^{-7}$ & $7.76 \times 10^{-4}$ & $4.57 \times 10^{-3}$ & $1.08 \times 10^{+0}$ & $7.86 \times 10^{-1}$ \\
\hline $\mathrm{F} 12$ & $1.09 \times 10^{-3}$ & $4.86 \times 10^{-7}$ & $6.97 \times 10^{-4}$ & $4.38 \times 10^{-3}$ & $1.23 \times 10^{+0}$ & $1.44 \times 10^{+0}$ \\
\hline $\mathrm{I}+\mathrm{F}$ & $1.29 \times 10^{-3}$ & $6.02 \times 10^{-7}$ & $7.76 \times 10^{-4}$ & $4.56 \times 10^{-3}$ & $1.02 \times 10^{+0}$ & $5.69 \times 10^{-1}$ \\
\hline \multicolumn{7}{|c|}{ H II regions } \\
\hline $\mathrm{I} 12$ & $1.77 \times 10^{-5}$ & $2.99 \times 10^{-8}$ & $1.73 \times 10^{-4}$ & $1.24 \times 10^{-2}$ & $3.11 \times 10^{+1}$ & $1.51 \times 10^{+3}$ \\
\hline F123 & $4.85 \times 10^{-4}$ & $3.69 \times 10^{-7}$ & $6.07 \times 10^{-4}$ & $8.04 \times 10^{-3}$ & $1.83 \times 10^{+0}$ & $4.34 \times 10^{+0}$ \\
\hline $\mathrm{F} 12$ & $4.19 \times 10^{-4}$ & $2.97 \times 10^{-7}$ & $5.45 \times 10^{-4}$ & $7.74 \times 10^{-3}$ & $2.09 \times 10^{+0}$ & $6.13 \times 10^{+0}$ \\
\hline $\mathrm{I}+\mathrm{F}$ & $5.03 \times 10^{-4}$ & $4.04 \times 10^{-7}$ & $6.36 \times 10^{-4}$ & $1.41 \times 10^{-2}$ & $2.32 \times 10^{+0}$ & $1.42 \times 10^{+1}$ \\
\hline \multicolumn{7}{|c|}{ Sum of the above components } \\
\hline $\mathrm{I} 12$ & $5.67 \times 10^{-4}$ & $3.47 \times 10^{-6}$ & $1.86 \times 10^{-3}$ & $6.51 \times 10^{-2}$ & $9.18 \times 10^{+0}$ & $1.24 \times 10^{+2}$ \\
\hline F123 & $1.59 \times 10^{-2}$ & $2.20 \times 10^{-4}$ & $1.48 \times 10^{-2}$ & $1.10 \times 10^{-1}$ & $1.70 \times 10^{+0}$ & $3.77 \times 10^{+0}$ \\
\hline F12 & $1.39 \times 10^{-2}$ & $1.85 \times 10^{-4}$ & $1.36 \times 10^{-2}$ & $1.11 \times 10^{-1}$ & $1.97 \times 10^{+0}$ & $5.52 \times 10^{+0}$ \\
\hline $\mathrm{I}+\mathrm{F}$ & $1.65 \times 10^{-2}$ & $2.25 \times 10^{-4}$ & $1.50 \times 10^{-2}$ & $1.10 \times 10^{-1}$ & $1.61 \times 10^{+0}$ & $3.36 \times 10^{+0}$ \\
\hline \multicolumn{7}{|c|}{ WMAP at $94 \mathrm{GHz}$} \\
\hline F123 & $2.75 \times 10^{-2}$ & $3.37 \times 10^{-4}$ & $1.83 \times 10^{-2}$ & $1.51 \times 10^{-1}$ & $1.33 \times 10^{+0}$ & $3.00 \times 10^{+0}$ \\
\hline
\end{tabular}

Table 5. The same as in Table 1, but for the indicated simulated beam.

\begin{tabular}{|c|c|c|c|c|c|c|}
\hline \multicolumn{7}{|c|}{ LFI4 4B } \\
\hline Beam & & & & & Skewness & Kurtosis \\
\hline Region & Average & Variance & $\mathrm{rms}$ & Peak-to-peak & Index & Index \\
\hline \multicolumn{7}{|c|}{ Dust + "correlated" diffuse free-free emission } \\
\hline $\mathrm{I} 12$ & $1.28 \times 10^{-2}$ & $1.97 \times 10^{-3}$ & $4.44 \times 10^{-2}$ & $1.46 \times 10^{+0}$ & $9.80 \times 10^{+0}$ & $1.42 \times 10^{+2}$ \\
\hline F12 & $1.68 \times 10^{-1}$ & $3.64 \times 10^{-2}$ & $1.91 \times 10^{-1}$ & $1.51 \times 10^{+0}$ & $2.39 \times 10^{+0}$ & $7.90 \times 10^{+0}$ \\
\hline $\mathrm{I}+\mathrm{F}$ & $1.81 \times 10^{-1}$ & $3.86 \times 10^{-2}$ & $1.96 \times 10^{-1}$ & $1.58 \times 10^{+0}$ & $2.21 \times 10^{+0}$ & $6.71 \times 10^{+0}$ \\
\hline \multicolumn{7}{|c|}{ Diffuse synchrotron emission } \\
\hline $\mathrm{I} 12$ & $1.17 \times 10^{-3}$ & $3.33 \times 10^{-6}$ & $1.82 \times 10^{-3}$ & $2.80 \times 10^{-2}$ & $6.80 \times 10^{+0}$ & $6.11 \times 10^{+1}$ \\
\hline F12 & $1.47 \times 10^{-2}$ & $1.14 \times 10^{-4}$ & $1.07 \times 10^{-2}$ & $6.64 \times 10^{-2}$ & $1.50 \times 10^{+0}$ & $2.39 \times 10^{+0}$ \\
\hline $\mathrm{I}+\mathrm{F}$ & $1.59 \times 10^{-2}$ & $1.18 \times 10^{-4}$ & $1.09 \times 10^{-2}$ & $6.60 \times 10^{-2}$ & $1.38 \times 10^{+0}$ & $1.86 \times 10^{+0}$ \\
\hline \multicolumn{7}{|c|}{ H II regions } \\
\hline $\mathrm{I} 12$ & $4.53 \times 10^{-4}$ & $2.17 \times 10^{-5}$ & $4.65 \times 10^{-3}$ & $3.89 \times 10^{-1}$ & $3.24 \times 10^{+1}$ & $1.73 \times 10^{+3}$ \\
\hline $\mathrm{F} 12$ & $5.48 \times 10^{-3}$ & $6.43 \times 10^{-5}$ & $8.02 \times 10^{-3}$ & $6.53 \times 10^{-2}$ & $2.50 \times 10^{+0}$ & $8.11 \times 10^{+0}$ \\
\hline $\mathrm{I}+\mathrm{F}$ & $5.93 \times 10^{-3}$ & $8.78 \times 10^{-5}$ & $9.37 \times 10^{-3}$ & $4.11 \times 10^{-1}$ & $5.89 \times 10^{+0}$ & $1.25 \times 10^{+2}$ \\
\hline \multicolumn{7}{|c|}{ Sum of the above components } \\
\hline $\mathrm{I} 12$ & $1.44 \times 10^{-2}$ & $2.38 \times 10^{-3}$ & $4.88 \times 10^{-2}$ & $1.65 \times 10^{+0}$ & $9.81 \times 10^{+0}$ & $1.45 \times 10^{+2}$ \\
\hline $\mathrm{F} 12$ & $1.89 \times 10^{-1}$ & $4.35 \times 10^{-2}$ & $2.09 \times 10^{-1}$ & $1.63 \times 10^{+0}$ & $2.34 \times 10^{+0}$ & $7.53 \times 10^{+0}$ \\
\hline $\mathrm{I}+\mathrm{F}$ & $2.03 \times 10^{-1}$ & $4.63 \times 10^{-2}$ & $2.15 \times 10^{-1}$ & $1.79 \times 10^{+0}$ & $2.17 \times 10^{+0}$ & $6.41 \times 10^{+0}$ \\
\hline
\end{tabular}


Table 6. The same as in Table 1, but for the indicated simulated beam.

\begin{tabular}{|c|c|c|c|c|c|c|}
\hline \multicolumn{7}{|c|}{ LFI4 4C } \\
\hline Beam & & & & & Skewness & Kurtosis \\
\hline Region & Average & Variance & rms & Peak-to-peak & Index & Index \\
\hline \multicolumn{7}{|c|}{ Dust + "correlated" diffuse free-free emission } \\
\hline I12 & $9.56 \times 10^{-3}$ & $1.12 \times 10^{-3}$ & $3.34 \times 10^{-2}$ & $1.13 \times 10^{+0}$ & $9.96 \times 10^{+0}$ & $1.49 \times 10^{+2}$ \\
\hline F12 & $1.08 \times 10^{-1}$ & $1.64 \times 10^{-2}$ & $1.28 \times 10^{-1}$ & $1.03 \times 10^{+0}$ & $2.55 \times 10^{+0}$ & $8.77 \times 10^{+0}$ \\
\hline $\mathrm{I}+\mathrm{F}$ & $1.17 \times 10^{-1}$ & $1.76 \times 10^{-2}$ & $1.33 \times 10^{-1}$ & $1.20 \times 10^{+0}$ & $2.36 \times 10^{+0}$ & $7.43 \times 10^{+0}$ \\
\hline \multicolumn{7}{|c|}{ Diffuse synchrotron emission } \\
\hline I12 & $8.75 \times 10^{-4}$ & $1.87 \times 10^{-6}$ & $1.37 \times 10^{-3}$ & $2.11 \times 10^{-2}$ & $6.81 \times 10^{+0}$ & $6.14 \times 10^{+1}$ \\
\hline $\mathrm{F} 12$ & $9.43 \times 10^{-3}$ & $5.01 \times 10^{-5}$ & $7.08 \times 10^{-3}$ & $4.49 \times 10^{-2}$ & $1.64 \times 10^{+0}$ & $2.90 \times 10^{+0}$ \\
\hline $\mathrm{I}+\mathrm{F}$ & $1.03 \times 10^{-2}$ & $5.21 \times 10^{-5}$ & $7.22 \times 10^{-3}$ & $4.46 \times 10^{-2}$ & $1.49 \times 10^{+0}$ & $2.27 \times 10^{+0}$ \\
\hline \multicolumn{7}{|c|}{ H II regions } \\
\hline $\mathrm{I} 12$ & $3.40 \times 10^{-4}$ & $1.24 \times 10^{-5}$ & $3.53 \times 10^{-3}$ & $2.93 \times 10^{-1}$ & $3.24 \times 10^{+1}$ & $1.71 \times 10^{+3}$ \\
\hline $\mathrm{F} 12$ & $3.51 \times 10^{-3}$ & $2.93 \times 10^{-5}$ & $5.41 \times 10^{-3}$ & $4.55 \times 10^{-2}$ & $2.69 \times 10^{+0}$ & $9.26 \times 10^{+0}$ \\
\hline $\mathrm{I}+\mathrm{F}$ & $3.85 \times 10^{-3}$ & $4.25 \times 10^{-5}$ & $6.52 \times 10^{-3}$ & $3.08 \times 10^{-1}$ & $7.07 \times 10^{+0}$ & $1.71 \times 10^{+2}$ \\
\hline \multicolumn{7}{|c|}{ Sum of the above components } \\
\hline $\mathrm{I} 12$ & $1.08 \times 10^{-2}$ & $1.35 \times 10^{-3}$ & $3.67 \times 10^{-2}$ & $1.27 \times 10^{+0}$ & $9.98 \times 10^{+0}$ & $1.52 \times 10^{+2}$ \\
\hline $\mathrm{F} 12$ & $1.21 \times 10^{-1}$ & $1.96 \times 10^{-2}$ & $1.40 \times 10^{-1}$ & $1.12 \times 10^{+0}$ & $2.50 \times 10^{+0}$ & $8.38 \times 10^{+0}$ \\
\hline $\mathrm{I}+\mathrm{F}$ & $1.32 \times 10^{-1}$ & $2.11 \times 10^{-2}$ & $1.45 \times 10^{-1}$ & $1.35 \times 10^{+0}$ & $2.32 \times 10^{+0}$ & $7.13 \times 10^{+0}$ \\
\hline
\end{tabular}

Table 7. The same as in Table 1, but for the indicated simulated beam.

\begin{tabular}{|c|c|c|c|c|c|c|}
\hline \multicolumn{7}{|c|}{ LFI4 4D } \\
\hline Beam & & & & & Skewness & Kurtosis \\
\hline Region & Average & Variance & $\mathrm{rms}$ & Peak-to-peak & Index & Index \\
\hline \multicolumn{7}{|c|}{ Dust + "correlated" diffuse free-free emission } \\
\hline $\mathrm{I} 12$ & $1.19 \times 10^{-2}$ & $1.73 \times 10^{-3}$ & $4.16 \times 10^{-2}$ & $1.41 \times 10^{+0}$ & $9.93 \times 10^{+0}$ & $1.48 \times 10^{+2}$ \\
\hline F12 & $1.47 \times 10^{-1}$ & $3.10 \times 10^{-2}$ & $1.76 \times 10^{-1}$ & $1.43 \times 10^{+0}$ & $2.60 \times 10^{+0}$ & $9.10 \times 10^{+0}$ \\
\hline $\mathrm{I}+\mathrm{F}$ & $1.59 \times 10^{-1}$ & $3.28 \times 10^{-2}$ & $1.81 \times 10^{-1}$ & $1.50 \times 10^{+0}$ & $2.41 \times 10^{+0}$ & $7.82 \times 10^{+0}$ \\
\hline \multicolumn{7}{|c|}{ Diffuse synchrotron emission } \\
\hline $\mathrm{I} 12$ & $1.09 \times 10^{-3}$ & $2.91 \times 10^{-6}$ & $1.71 \times 10^{-3}$ & $2.62 \times 10^{-2}$ & $6.81 \times 10^{+0}$ & $6.13 \times 10^{+1}$ \\
\hline F12 & $1.28 \times 10^{-2}$ & $9.39 \times 10^{-5}$ & $9.69 \times 10^{-3}$ & $6.22 \times 10^{-2}$ & $1.66 \times 10^{+0}$ & $3.04 \times 10^{+0}$ \\
\hline $\mathrm{I}+\mathrm{F}$ & $1.39 \times 10^{-2}$ & $9.70 \times 10^{-5}$ & $9.85 \times 10^{-3}$ & $6.17 \times 10^{-2}$ & $1.53 \times 10^{+0}$ & $2.46 \times 10^{+0}$ \\
\hline \multicolumn{7}{|c|}{ H II regions } \\
\hline $\mathrm{I} 12$ & $4.24 \times 10^{-4}$ & $1.94 \times 10^{-5}$ & $4.40 \times 10^{-3}$ & $3.67 \times 10^{-1}$ & $3.27 \times 10^{+1}$ & $1.75 \times 10^{+3}$ \\
\hline F12 & $4.80 \times 10^{-3}$ & $5.55 \times 10^{-5}$ & $7.45 \times 10^{-3}$ & $6.36 \times 10^{-2}$ & $2.73 \times 10^{+0}$ & $9.59 \times 10^{+0}$ \\
\hline $\mathrm{I}+\mathrm{F}$ & $5.22 \times 10^{-3}$ & $7.61 \times 10^{-5}$ & $8.73 \times 10^{-3}$ & $3.88 \times 10^{-1}$ & $6.26 \times 10^{+0}$ & $1.35 \times 10^{+2}$ \\
\hline \multicolumn{7}{|c|}{ Sum of the above components } \\
\hline $\mathrm{I} 12$ & $1.34 \times 10^{-2}$ & $2.09 \times 10^{-3}$ & $4.57 \times 10^{-2}$ & $1.58 \times 10^{+0}$ & $9.96 \times 10^{+0}$ & $1.52 \times 10^{+2}$ \\
\hline F12 & $1.65 \times 10^{-1}$ & $3.70 \times 10^{-2}$ & $1.92 \times 10^{-1}$ & $1.56 \times 10^{+0}$ & $2.55 \times 10^{+0}$ & $8.70 \times 10^{+0}$ \\
\hline $\mathrm{I}+\mathrm{F}$ & $1.78 \times 10^{-1}$ & $3.92 \times 10^{-2}$ & $1.98 \times 10^{-1}$ & $1.69 \times 10^{+0}$ & $2.37 \times 10^{+0}$ & $7.49 \times 10^{+0}$ \\
\hline
\end{tabular}

recovery but also a detailed imaging of the last scattering surface and a detailed study of the all information, cosmological and astrophysical, contained in the frequency maps. Therefore, in order to avoid spurious signals at a level comparable with the PLANCK sensitivity, it is required that the contamination from systematic effects does not cross the threshold of a few $\mu \mathrm{K}$.
Among the set of analyzed optical configurations we can identify a subsample that reaches a good trade-off between the angular resolution (see Table 2 of Paper I) and the suppression of the straylight contamination.

In the case of LFI9 the configuration 9B shows a global peak-to-peak (rms) GSC less than about $2 \mu \mathrm{K}(0.2 \mu \mathrm{K})$ simultaneously reaching an angular resolution $(F W H M)$ of $10.02^{\prime}$. 
Table 8. rms and peak-to-peak value of the simulated TOD (antenna temperature expressed in $\mu \mathrm{K}$ ) as functions of the spillover from intermediate, far beam, and both the intermediate/far beam region for all simulated beams obtained making use of the sum of the three considered templates of Galactic templates. We neglect here the contribution from the third-order optical interactions.

\begin{tabular}{cccccccc}
\hline \hline & \multicolumn{7}{c}{ Summary table } \\
Beam & Spillover & I & I & F & F & I + F & I + F \\
& $\%$ & rms & Peak-to-peak & rms & Peak-to-peak & rms & Peak-to-peak \\
\hline LFI9 9A & 0.31 & $8.38 \times 10^{-3}$ & $2.94 \times 10^{-1}$ & $7.33 \times 10^{-2}$ & $6.16 \times 10^{-1}$ & $7.61 \times 10^{-2}$ & $6.38 \times 10^{-1}$ \\
LFI9 9B & 0.68 & $2.78 \times 10^{-2}$ & $8.84 \times 10^{-1}$ & $1.90 \times 10^{-1}$ & $1.87 \times 10^{+0}$ & $2.00 \times 10^{-1}$ & $1.92 \times 10^{+0}$ \\
LFI9 9C & 1.90 & $8.08 \times 10^{-2}$ & $2.26 \times 10^{+0}$ & $6.72 \times 10^{-1}$ & $6.18 \times 10^{+0}$ & $7.07 \times 10^{-1}$ & $6.35 \times 10^{+0}$ \\
LFI4 4A & 0.10 & $1.86 \times 10^{-3}$ & $6.51 \times 10^{-2}$ & $1.36 \times 10^{-2}$ & $1.11 \times 10^{-1}$ & $1.50 \times 10^{-2}$ & $1.10 \times 10^{-1}$ \\
LFI4 4B & 1.31 & $4.88 \times 10^{-2}$ & $1.65 \times 10^{+0}$ & $2.09 \times 10^{-1}$ & $1.63 \times 10^{+0}$ & $2.15 \times 10^{-1}$ & $1.79 \times 10^{+0}$ \\
LFI4 4C & 0.87 & $3.67 \times 10^{-2}$ & $1.27 \times 10^{+0}$ & $1.40 \times 10^{-1}$ & $1.12 \times 10^{+0}$ & $1.45 \times 10^{-1}$ & $1.35 \times 10^{+0}$ \\
LFI4 4D & 1.15 & $4.57 \times 10^{-2}$ & $1.58 \times 10^{+0}$ & $1.92 \times 10^{-1}$ & $1.56 \times 10^{+0}$ & $1.98 \times 10^{-1}$ & $1.69 \times 10^{+0}$ \\
\hline
\end{tabular}

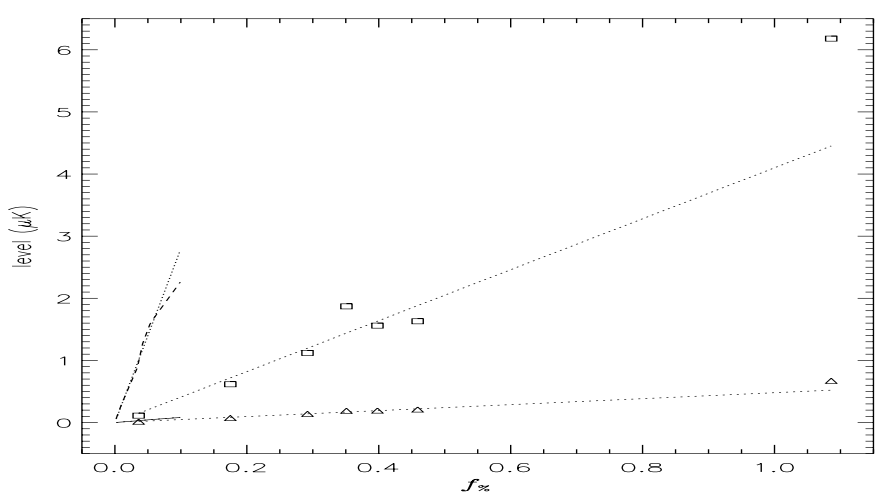

Fig. 7. Dependence of the rms (solid line for the intermediate beam and triangles for the far beam) and the peak-to-peak values (dashed line for the intermediate beam and squares for the far beam) of the GSC at $100 \mathrm{GHz}$ on the (per cent) fractional contribution to the integrated beam response from the considered beam region and corresponding linear approximations described by Eqs. (3)-(6) (dotted lines).

Configuration 9A shows a lower GSC, but with a worse angular resolution $\left(10.56^{\prime}\right)$, while configuration 9C makes it possible to reach a resolution of $9.54^{\prime}$ but introduces a relatively large GSC, as its peak-to-peak (rms) signal is of about $7 \mu \mathrm{K}(0.7 \mu \mathrm{K})$. The worse resolution for LFI4 with respect to LFI9 is due to the different location of the corresponding feed on the focal surface (see Mandolesi et al. 2000). On the other hand, our analysis identifies a well defined optical configuration $(4 \mathrm{C})$ that makes it possible to reach the best angular resolution $\left(12.08^{\prime}\right)$ with the minimum GSC, i.e. a peak-to-peak (rms) signal less than about $1.5 \mu \mathrm{K}(0.15 \mu \mathrm{K})$.

In Appendix B we also report the results obtained for a simulation at $30 \mathrm{GHz}$. We find some qualitative differences between the GSC at these two frequencies, related to their optical behaviour and to the different role of the Galactic foreground.

An accurate computation of the GSC in PLANCK polarization data could in principle be carried out using the formalism described in Challinor et al. (2000). On the other hand, since no microwave polarization surveys are currently available, a detailed computation based on current simulated templates (see e.g. Giardino et al. 2002) may provide only indicative results.
A first order analysis aimed at obtaining a robust upper limit on the rms GSC in the PLANCK LFI polarization data can be obtained with a simple argument. The signals, $I_{i j}(i=1,2)$, in the four radiometers associated to a couple of LFI feeds symmetrically located in the focal plane can be expressed (in terms of antenna temperature) as:

$$
\begin{aligned}
& 2 I_{11}=T+\delta T_{11}^{\mathrm{s}}+\left(Q+\delta Q_{11}^{\mathrm{s}}\right) \cos \left(2 \phi_{11}\right)+\left(U+\delta U_{11}^{\mathrm{s}}\right) \sin \left(2 \phi_{11}\right) \\
& 2 I_{12}=T+\delta T_{12}^{\mathrm{s}}-\left(Q+\delta Q_{12}^{\mathrm{s}}\right) \cos \left(2 \phi_{11}\right)-\left(U+\delta U_{12}^{\mathrm{s}}\right) \sin \left(2 \phi_{11}\right) \\
& 2 I_{21}=T+\delta T_{21}^{\mathrm{s}}+\left(Q+\delta Q_{21}^{\mathrm{s}}\right) \cos \left(2 \phi_{21}\right)+\left(U+\delta U_{21}^{\mathrm{s}}\right) \sin \left(2 \phi_{21}\right) \\
& 2 I_{22}=T+\delta T_{22}^{\mathrm{s}}-\left(Q+\delta Q_{22}^{\mathrm{s}}\right) \cos \left(2 \phi_{21}\right)-\left(U+\delta U_{22}^{\mathrm{s}}\right) \sin \left(2 \phi_{21}\right) .
\end{aligned}
$$

Here $\phi_{21}=\phi_{11}+\pi / 4, \phi_{11}$ is the angle between the axis $x_{\mathrm{bf}}$ of the beam frame corresponding to the first feed and the direction of the parallel in the considered pointing direction, $T, Q$ and $U$ are the (intensity and linear polarization) Stokes parameters in the main beam, and the terms $\delta T_{i j}^{\mathrm{s}}, \delta Q_{i j}^{\mathrm{s}}, \delta U_{i j}^{\mathrm{s}}$ account for the spurious contributions due to the Galactic straylight. For each pointing direction they are different for the four radiometers because of the different level and orientation of the corresponding beams. The measurement of the Stokes parameters $Q$ and $U$ is obtained by combining the signals in the four radiometers: $Q=\left(I_{11}-I_{12}\right) \cos \left(2 \phi_{11}\right)-\left(I_{21}-I_{22}\right) \sin \left(2 \phi_{11}\right)$, $U=\left(I_{21}-I_{22}\right) \cos \left(2 \phi_{11}\right)+\left(I_{11}-I_{22}\right) \sin \left(2 \phi_{11}\right)$. The Galactic synchrotron emission is partially polarized $(\sim 30 \%)$, while the Galactic free-free and thermal dust emission, dominant at $v \gtrsim$ $50 \mathrm{GHz}$, are only weakly polarized (a few \%). Therefore, at least in the cosmological window, a GSC relevant for the polarization measure mainly derives from the differences between the temperature straylight signals, $\delta T_{i j}^{\mathrm{s}}$, in each pair of radiometers associated to the same feed. Assuming a typical value $\sim 1 / 2$ for $\cos \left(2 \phi_{11}\right)$ and $\sin \left(2 \phi_{11}\right)$, we find an rms GSC on $Q$ and $U$ similar to that found above for $T$ (see Eqs. (4) and (6)), to be considered as a pessimistic upper limit, corresponding to a difference of a factor $\simeq 2$ in the temperature straylight signals in each pair of radiometers.

We then conclude that, at least in terms of rms and in the cosmological window, the suppression of the GSC in the polarization data will be at least at the same level of that achieved for the GSC in the temperature data. 


\section{Discussion and conclusions}

Satellite CMB anisotropy missions, such as WMAP and PLANCK, and also the new generation of balloon-borne and ground experiments, make use of complex multi-frequency instruments in the focal surface of a meter class telescope to allow the joint study of CMB and foreground anisotropies, necessary for a high quality component separation. In the so-called "cosmological window", between $\sim 70 \mathrm{GHz}$ and $\sim 300 \mathrm{GHz}$, where foreground contamination is minimal, it is extremely important to reach the best angular resolution (necessary to measure the high order acoustic peaks of CMB anisotropy) keeping at the same time the straylight contamination at acceptable levels (peak-to-peak value of a few $\mu \mathrm{K}$ ).

Focusing, as a working case, on the $100 \mathrm{GHz}$ channels of PLANCK LFI, we have presented extensive simulations of the straylight contamination starting from the set of simulated optical configurations described in Paper I, in order to find the best compromise between resolution and GSC.

Adopting some templates of Galactic foreground extrapolated from radio, IR, and $\mathrm{H} \alpha$ surveys we found that it is possible to improve the angular resolution by about 5-7\% and to reach for example $10^{\prime}-12^{\prime} F W H M$ at $100 \mathrm{GHz}$ by keeping the overall straylight contamination below the level of a few $\mu \mathrm{K}$ in terms of peak-to-peak value and about 10 times smaller in terms of rms, which is necessary to avoid systematic errors comparable with the PLANCK sensitivity.

We compared the level of straylight introduced by the different Galactic components for different beam regions and provided simple approximate relations giving the rms and peakto-peak levels of the GSC for the intermediate and far beam as functions of the corresponding contributions to the integrated beam response, related to the spillover.

For the considered optical designs, the most important straylight contamination derives from the far beam, where the Galactic signal overwhelms the other straylight contributions (nevertheless, in the intermediate beam, the straylight contamination from CMB fluctuations is found to be non-negligible compared to the GSC).

We demonstrated that including the third order optical interactions changes the results of straylight analysis only by some per cent ( $\$ 5 \%$ in terms of rms and peak-to-peak values). As discussed in Paper I, this is extremely important for optical design optimization studies, proving that accurate enough optical simulations can be carried out saving about $75 \%$ of the computational time without a relevant loss of accuracy.

Keeping the GSC in the temperature data at very low level directly assures a similar suppression of the GSC in the polarization data, at least in terms of rms. Burigana et al. (2001) found that the angular power spectrum (expressed in terms of $\left.\ell(2 \ell+1) C_{\ell} / 4 \pi\right)$ of the GSC from the far beam is almost independent of the possible exclusion of low Galactic latitude regions in the data analysis and shows only a moderate dependence on the multipole $\ell$ up to $\ell \approx 10^{2}$, where it likely decreases because of the far beam smoothing at degree and sub-degree scales. By simply assuming $\ell(2 \ell+1) C_{\ell} / 4 \pi \simeq$ const. and remembering the usual relation $\mathrm{rms} \simeq \sum_{\ell_{\min }}^{\ell_{\max }}(2 \ell+$ 1) $C_{\ell} / 4 \pi$, the rms of the GSC can then be approximated as $\mathrm{rms} \simeq \sum_{\ell_{\min }}^{\ell_{\max }}($ const. $/ \ell) \simeq$ const. $\times \int_{\ell_{\min }}^{\ell_{\max }} \mathrm{d} \ell / \ell$. By taking $\ell_{\min }=1$, we have rms $\simeq 4.6 \times$ const. $\times \ln \left(\ell_{\max }\right) / \ln (100)$. The (conservative) upper limit of $0.2 \mu \mathrm{K}$ to the rms value of the GSC from the far beam derived in the previous section then implies const. $\simeq \ell(2 \ell+1) C_{\ell} / 4 \pi \lesssim 8.7 \times 10^{-3} \mu \mathrm{K}^{2}$ corresponding to const. $\lesssim 1.4 \times 10^{-2} \mu \mathrm{K}^{2}$ when expressed in terms of thermodynamic temperatures for an easier comparison with the $\mathrm{CMB}\left(\mathrm{rms}_{\mathrm{th}}^{2} \simeq 1.66 \mathrm{rms}_{\text {ant }}^{2} \text { at } 100 \mathrm{GHz}\right)^{13}$. The estimate of the impact of the GSC on the determination of the CMB polarization modes is uncertain because of the current poor observational status on CMB polarization. We will assume typical values predicted on the basis of current WMAP results (Spergel et al. 2003) for numerical estimates. The above value of $\sim 1.4 \times 10^{-2} \mu \mathrm{K}^{2}$ is significantly lower (by a factor $\simeq 5$ ) than the power of the $E$ mode expected at multipoles close to the (reionization) bump at $\ell \sim 5$ and at multipoles $\ell \gtrsim 50$, when the $E$ mode increases up to its first peak. On the other hand, although the direct Galactic foreground contamination, if not accurately separated, may present a problem more critical than the GSC, this level of GSC may represent a serious limitation for the accurate measure of the $E$ mode at $\ell \sim 10-30$ (where it is expected to show a minimum $\sim 10^{-2} \mu \mathrm{K}^{2}$ ), of the $B$ mode (expected to assume values $\gtrsim 2.5 \times 10^{-3} \mu \mathrm{K}^{2}$ with possibly bumps $\left.\sim(1-4) \times 10^{-2} \mu \mathrm{K}^{2}\right)$ and of the $E T$ mode at some particular multipoles (close to its changes of sign, where it obviously tends to vanish). At low multipoles, an accurate data analysis, possibly based on precise ground beam measures and in-flight recovery, is then required to subtract this effect (of course, this is also necessary for accurate polarization imaging). At multipoles larger then a few tens, a cross-check of the results obtained by analyzing nearly the whole sky with those derived by considering selected (but sufficiently large) sky areas having simultaneously low Galactic emission and low GSC can significantly alleviate this problem ${ }^{14}$. While further investigations are needed, simulations like those presented here combined with the improvement in the mapping of Galactic foreground (in both temperature and polarization) represent the input necessary for this work.

Finally, we have compared the results at $100 \mathrm{GHz}$ with those found at $30 \mathrm{GHz}$, where the GSC is more critical, showing a peak-to-peak value at a level of $\simeq 5-7 \mu \mathrm{K}$. In comparison with previous analyses (see e.g. Burigana et al. 2001), this represents a clear improvement in straylight rejection related to the

13 This upper limit obtained with a simple heuristic argument has been confirmed by a detailed computation of the angular power spectrum of the GSC from the far beam in the representative case of the configuration LFI9 9B: by considering the map of correlated dust and free-free emission, in the multipole range $1 \leq \ell \leq 95$ we find $10^{-3} \mu \mathrm{K}^{2} \lesssim \ell(2 \ell+1) C_{\ell} / 4 \pi \lesssim 1.6 \times 10^{-2} \mu \mathrm{K}^{2}$ (in terms of thermodynamic temperature) with an average value over the considered multipole range (over the range $1 \leq \ell \leq 10$ or $11 \leq \ell \leq 95$ ) of $\simeq 3 \times 10^{-3} \mu \mathrm{K}^{2}$ $\left(\simeq 8 \times 10^{-3} \mu \mathrm{K}^{2}\right.$ or $\left.\simeq 2.5 \times 10^{-3} \mu \mathrm{K}^{2}\right)$.

14 For example, for the configuration 9B of LFI9, we find that excluding the fraction (only $\sim 10 \%$ ) of the TOD with a GSC signal (in antenna temperature) larger than $0.4 \mu \mathrm{K}$ (a value $\sim 2$ times larger than the GSC rms found) implies a reduction of a factor $\sim 2$ of the $\mathrm{rms}-\mathrm{a}$ factor $\sim 4$ in terms of $C_{\ell}$ - of the TOD effectively used with respect to the value of the complete set of TOD. 
optimization of the overall PLANCK optical scheme (Dubruel et al. 2000). Clearly, assuming different Galactic templates implies differences in the computed straylight signals. On the other hand, even for the quite different input maps adopted in these tests, differences larger than $\simeq 1-2 \mu \mathrm{K}$ are limited to quite localized regions, close to the Galactic plane, where the CMB anisotropy is dominated by the very high Galactic signal in the main beam. This suggests that, even far from the "cosmological window", a subtraction of the GSC well down to the $\sim 1 \mu \mathrm{K}$ level could be obtained by evaluating with few iterations the straylight contamination signal for the maps directly derived from PLANCK observations, provided that the antenna beam response could be quite accurately modelled.

Acknowledgements. It is a pleasure to thank C. L. Bennett for useful explanations on WMAP 1-yr data products and J. P. Leahy and L. A. Popa for interesting comments. Some of the results in this paper have been derived using HEALPix (Gòrski et al. 1999). We warmly thank J. M. Lamarre for constructive comments and suggestions and for the careful reading of the first version of this work.

\section{References}

Barnes, C., Hill, R. S., Hinshaw, G., et al. 2003, ApJS, 148, 151 Bennett, C. L., Banday, A. J., Gòrski, K. M., et al. 1996, ApJ, 464, L1

Bennett, C. L., Halpern, M., Hinshaw, G., et al. 2003a, ApJS, 148, 1 Bennett, C. L., Hill, R. S., Hinshaw, G., et al. 2003b, ApJS, 148, 97

Bernard, J. P., Puget, J. L., Sygnet, J. F., \& Lamarre, J. M. 2002, Draft note on HFI's view on Planck Scanning Strategy, Technical Note PL-HFI-IAS-TN-SCAN01, 0.2.1

Bersanelli, M., et al. 1996, ESA, COBRAS/SAMBA Report on the Phase A Study, D/SCI(96)3

Bersanelli, M., Maino, D., \& Mennella, A. 2002, Riv. Nuovo Cimento, 25,9

Bouchet, F. R., \& Gispert, R. 1999, NA, 4, 443

Burigana, C., Malaspina, M., Mandolesi, N., et al. 1997, Int. Rep. TeSRE/CNR 198/1997, November [arXiv: astro-ph/9906360]

Burigana, C., Maino, D., Mandolesi, N., et al. 1998, A\&AS, 130, 551

Burigana, C., Maino, D., Natoli, P., et al. 2000, Straylight effects and beam reconstruction in Planck/LFI observations, talk at the Planck Scanning Strategy Meeting, Villa Mondragone - Monteporzio Catone (Roma), Italy, 22-23 June 2000

Burigana, C., Maino, D., Gòrski, K. M., et al. 2001, A\&A, 373, 345

Burigana, C., \& Sáez, D. 2003, A\&A, 409, 423

Challinor, A., Fosalba, P., Mortlock, D., et al. 2000, Phys. Rev. D, 65, 023505
De Maagt, P., Polegre, A. M., \& Crone, G. 1998, PlANCK - Straylight Evaluation of the Carrier Configuration, Technical Report ESA, PT-TN-05967, 1/0

De Zotti, G., Toffolatti, L., Argüeso Gómez, F., et al. 1999, Proc. of the EC-TMR Conference 3 K Cosmology, Roma, Italy, 5-10 October 1998, ed. L. Maiani, F. Melchiorri, \& N. Vittorio, AIP Conf. Proc., 476, 204

Dubruel, D., Cornut, M., Fargant, G., et al. 2000, in Millennium Conference on Antennas \& Propagation - 2000, ed. D. Danesy, \& H. Sawaya, ESA Conf. Proc., SP-444, European Space Agency, CD-ROM

Finkbeiner, D. P. 2003, ApJS, 146, 407

Giardino, G., Banday, A. J., Gòrski, K. M., et al. 2002, A\&A, 387, 82

Górski, K. M., Hivon, E., \& Wandelt, B. D. 1999, Proc. of the MPA/ESO Conference on Evolution of Large-Scale Structure: from Recombination to Garching, ed. A. J. Banday, R. K. Sheth, \& L. Da Costa, 37 [arXiv: astro-ph/9812350]

Haslam, C. G. T., Stoffel, H., Saltes, C. J., \& Wilson, W. E. 1982, A\&AS, 47, 1

Hinshaw, G., Barnes, C., Bennett, C. L., et al. 2003a, ApJS, 148, 63

Hinshaw, G., Spergel, D. N., Verde, L., et al. 2003b, ApJS, 148, 135

Kogut, A., Spergel, D. N., Barnes, C., et al. 2003, ApJS, 148, 161

Lamarre, J. M., Maffei, B., Ade, P. A. R., et al. 2002, in Experimental Cosmology at millimetre wavelengths - 2K1BC Workshop, Breuil-Cervinia (AO), Valle d'Aosta, Italy, 9-13 July 2001, ed. M. De Petris, \& M. Gervasi, AIP Conf. Proc., 616, 213

Maino, D., Burigana, C., Maltoni, M., et al. 1999, A\&AS, 140, 383

Maino, D., Farusi, A., Baccigalupi, C., et al. 2002, MNRAS, 334, 53

Mandolesi, N., et al. 1998, PLANCK LFI, A Proposal Submitted to the ESA

Mandolesi, N., Bersanelli, M., Burigana, C., et al. 2000, A\&AS, 145, 323

Mandolesi, N., Bersanelli, M., Butler, R. C., et al. 2002, in Experimental Cosmology at millimetre wavelengths - 2K1BC Workshop, Breuil-Cervinia (AO), Valle d'Aosta, Italy, 9-13 July 2001, ed. M. De Petris, \& M. Gervasi, AIP Conf. Proc., 616, 193

Netterfield, C. B., Ade, P. A. R., Bock, J. J., et al. 2002, ApJ, 571, 604

Page, L., Barnes, C., Hinshaw, G., et al. 2003, ApJS, 148, 39

Paladini, R., Burigana, C., Davies, R. D., et al. 2003, A\&A, 397, 213

Pryke, C., Halverson, N. W., Leitch, E. M., et al. 2002, ApJ, 568, 46

Puget, J. L., et al. 1998, HFI for the PlancK Mission, A Proposal Submitted to the ESA

Sandri, M., Villa, F., Nesti, R., et al. 2004, A\&A, 428, 299 (Paper I)

Schlegel, D. J., Finkbeiner, D. P., \& Davies, M. 1998, ApJ, 500, 525

Spergel, D. N., Verde, L., Peiris, H. V., et al. 2003, ApJS, 148, 175

Stompor, R., Abroe, M., Ade, P., et al. 2001, ApJ, 561, L7

Tauber, J. A. 2001, in The Extragalactic Infrared Background and its Cosmological Implications, ed. M. Harwit, Proc. IAU Symp., 204, 493 


\section{Online Material}




\section{Appendix A: Transformation rules between telescope frame and beam frame}

Let $s$ be the unit vector, chosen outward the Sun direction, of the spin axis direction and $\hat{k}$ that of the direction, $z$, of the telescope line of sight (LOS), pointing at an angle $\alpha \sim 85^{\circ}$ from the direction of $s$.

In the plane tangent to the celestial sphere in the direction of the LOS we choose two coordinates $x$ and $y$, respectively defined by the unit vector $\hat{i}$ and $\hat{j}$ according to the convention that the unit vector $\hat{i}$ always points toward $s$ and that $x, y, z$ is a standard Cartesian frame, called here as the telescope frame.

Let $\hat{i}_{\mathrm{bf}}, \hat{j}_{\mathrm{bf}}, \hat{k}_{\mathrm{bf}}$ be the unit vectors corresponding to the Cartesian axes $x_{\mathrm{bf}}, y_{\mathrm{bf}}, z_{\mathrm{bf}}$ of the beam frame; $\hat{k}_{\mathrm{bf}}$ defines the direction of the beam centre axis in the telescope frame. The beam frame is defined with respect to the telescope frame by three angles: $\theta_{\mathrm{B}}, \phi_{\mathrm{B}}, \psi_{\mathrm{B}}\left(\theta_{\mathrm{B}}\right.$ and $\phi_{\mathrm{B}}$, two standard polar coordinates defining the direction of the beam centre axis, range respectively from $0^{\circ}$, for an on-axis beam, to some degrees, for LFI off-axis beams, and from $0^{\circ}$ to $360^{\circ}$ ).

Let ${\hat{i^{\prime}}}_{\text {bf }}, \hat{j}_{\text {bf }}^{\prime},{\hat{k^{\prime}}}_{\mathrm{bf}^{\prime}}\left({\hat{k^{\prime}}}_{\mathrm{bf}}=\hat{k}_{\mathrm{bf}}\right)$ be the unit vectors corresponding to the Cartesian axes $x^{\prime}, y^{\prime}, z^{\prime}$ of an intermediate frame, defined by the two angles $\theta_{\mathrm{B}}$ and $\phi_{\mathrm{B}}$, obtained by the telescope frame $x, y, z$ when the unit vector of the axis $z$ is rotated by an angle $\theta_{\mathrm{B}}$ on the plane defined by the unit vector of the axis $z$ and the unit vector $\hat{k}_{\mathrm{bf}}$ up to reach $\hat{k}_{\mathrm{bf}}$ :

$$
\begin{aligned}
\hat{k}_{\mathrm{bf}}^{\prime}=\hat{k}_{\mathrm{bf}}= & \cos \left(\phi_{\mathrm{B}}\right) \sin \left(\theta_{\mathrm{B}}\right) \hat{i}+\sin \left(\phi_{\mathrm{B}}\right) \sin \left(\theta_{\mathrm{B}}\right) \hat{j} \\
& +\cos \left(\theta_{\mathrm{B}}\right) \hat{k}
\end{aligned}
$$

$$
\begin{aligned}
{\hat{i^{\prime}}}_{\mathrm{bf}}= & {\left[\cos \left(\phi_{\mathrm{B}}\right)^{2} \cos \left(\theta_{\mathrm{B}}\right)+\sin \left(\phi_{\mathrm{B}}\right)^{2}\right] \hat{i} } \\
& +\left[\sin \left(\phi_{\mathrm{B}}\right) \cos \left(\phi_{\mathrm{B}}\right)\left(\cos \left(\theta_{\mathrm{B}}\right)-1\right)\right] \hat{j} \\
& -\sin \left(\theta_{\mathrm{B}}\right) \cos \left(\phi_{\mathrm{B}}\right) \hat{k}
\end{aligned}
$$

$$
\begin{aligned}
\hat{j}_{\mathrm{bf}}^{\prime}= & {\left[\sin \left(\phi_{\mathrm{B}}\right) \cos \left(\phi_{\mathrm{B}}\right)\left(\cos \left(\theta_{\mathrm{B}}\right)-1\right)\right] \hat{i} } \\
& +\left[\cos \left(\theta_{\mathrm{B}}\right) \sin \left(\phi_{\mathrm{B}}\right)^{2}+\cos \left(\phi_{\mathrm{B}}\right)^{2}\right] \hat{j} \\
& -\sin \left(\theta_{\mathrm{B}}\right) \sin \left(\phi_{\mathrm{B}}\right) \hat{k} .
\end{aligned}
$$

The beam frame is obtained from the intermediate frame through a further (anti-clockwise) rotation of by angle $\psi_{\mathrm{B}}$ (ranging from $0^{\circ}$ to $360^{\circ 15}$ ) around $\hat{k}_{\mathrm{bf}}$ and is therefore explicitly given by:

$$
\begin{aligned}
\hat{i}_{\mathrm{bf}}= & {\left[\cos \left(\psi_{\mathrm{B}}\right){\hat{i^{\prime}}}_{\mathrm{bf}, x}+\sin \left(\psi_{\mathrm{B}}\right){\hat{j^{\prime}}}_{\mathrm{bf}, x}\right] \hat{i} } \\
& +\left[\cos \left(\psi_{\mathrm{B}}\right){\hat{i^{\prime}}}_{\mathrm{bf}, y}+\sin \left(\psi_{\mathrm{B}}\right){\hat{j^{\prime}}}_{\mathrm{bf}, y}\right] \hat{j} \\
& +\left[\cos \left(\psi_{\mathrm{B}}\right){\hat{i^{\prime}}}_{\mathrm{bf}, z}+\sin \left(\psi_{\mathrm{B}}\right){\hat{j^{\prime}}}_{\mathrm{bf}, z}\right] \hat{z} \\
\hat{j}_{\mathrm{bf}}= & {\left[-\sin \left(\psi_{\mathrm{B}}\right){\hat{i^{\prime}}}_{\mathrm{bf}, x}+\cos \left(\psi_{\mathrm{B}}\right){\hat{j^{\prime}}}_{\mathrm{bf}, x}\right] \hat{i} } \\
& +\left[-\sin \left(\psi_{\mathrm{B}}\right){\hat{i^{\prime}}}_{\mathrm{bf}, y}+\cos \left(\psi_{\mathrm{B}}\right){\hat{\hat{j}^{\prime}}}_{\mathrm{bf}, y}\right] \hat{j} \\
& +\left[-\sin \left(\psi_{\mathrm{B}}\right){\hat{i^{\prime}}}_{\mathrm{bf}, z}+\cos \left(\psi_{\mathrm{B}}\right){\hat{j^{\prime}}}_{\mathrm{bf}, z}\right] \hat{z},
\end{aligned}
$$

\footnotetext{
15 We note that, in other conventions, angles $\phi_{\mathrm{B}}^{\prime}$ and $\psi_{\mathrm{B}}^{\prime}$ ranging from $-180^{\circ}$ to $180^{\circ}$ are given, instead of $\phi_{\mathrm{B}}$ and $\psi_{\mathrm{B}}$. The angles $\phi_{\mathrm{B}}$ and $\psi_{\mathrm{B}}$ here defined are equal to $\phi_{\mathrm{B}}^{\prime}$ and $\psi_{\mathrm{B}}^{\prime}$ when they are positive and are given respectively by $360^{\circ}+\phi_{\mathrm{B}}^{\prime}$ and $360^{\circ}+\psi_{\mathrm{B}}^{\prime}$ for negative $\phi_{\mathrm{B}}^{\prime}$ and $\psi_{\mathrm{B}}^{\prime}$.
}

where the bottom index $x(y, z)$ indicates the component of the intermediate frame unit vector along the axis $x(y, z)$ of the telescope frame, as defined by Eqs. (A1)-(A3).

\section{Appendix B: Simulations at $30 \mathrm{GHz}$}

The feed horn at $30 \mathrm{GHz}$ considered in these simulations is specified by its Spherical Wave Expansion (SWE) provided by Alcatel Space Industries, since the subreflector is in the near field of the corrugated horn and near field effects cannot be neglected. The feed horn directivity is about $22 \mathrm{dBi}$, the ET is $30 \mathrm{~dB}$ at $22^{\circ}$, and the main beam has a $F W H M$ resolution of $33.73^{\prime}$. The beam position and orientation are identified by $\left(\theta_{\mathrm{B}}, \phi_{\mathrm{B}}, \psi_{\mathrm{B}}\right)=\left(4.3466^{\circ}, 153.6074^{\circ}, 337.5^{\circ}\right)$.

We carry out the simulation of PLANCK observations as described in Sect. 2 by assuming both the Galactic templates presented in Sect. 2.2 and the WMAP map at $33 \mathrm{GHz}$. The results are summarized in Table 7 while Fig. B.1 shows the TOD corresponding to the overall straylight signal (from the far plus intermediate beam) for the sum of the three Galactic components described in Sect. 2.2 and for the WMAP map. In spite of the differences in the foreground templates (the dominant signal deriving from Galactic diffuse free-free emission using the templates of Sect. 2.2, and, according to Bennett et al. $2003 \mathrm{~b}$, from Galactic diffuse synchrotron emission using the WMAP map) the peak-to-peak value is at a level of $\simeq 5-7 \mu \mathrm{K}$ for the contributions from both the far and intermediate beam (rms $\sim 1 \mu \mathrm{K}$, mainly due to the signal in the far beam). The right panel of Fig. B.1 reports shows the difference between the TOD obtained using these two different templates: only for $\simeq 0.01 \%(0.09,0.3,10 \%)$ of the samples of the TOD is the difference larger than $4 \mu \mathrm{K}(3,2,1 \mu \mathrm{K})$. The figure also shows that differences larger than $\simeq 1-2 \mu \mathrm{K}$ are localized quite close to the Galactic plane, where the CMB anisotropy is dominated by the very high Galactic signal in the main beam. This implies that a subtraction of the GSC down to the $\sim 1 \mu \mathrm{K}$ level does not require a particularly accurate description of the microwave sky emission nor particularly sophisticated computations, even at frequencies where the GSC is relevant, but is mainly related to a good knowledge of the beam response.

Clearly, the unsubtracted GSC is relevant at frequencies far from the "cosmological window". On the other hand, these results represent a significant optical improvement compared to the analyses of GSC at $30 \mathrm{GHz}$ by Burigana et al. (2001), based on the optical simulations by De Maagt et al. (1998), predicting a similar level of GSC from the far beam but a contamination from the intermediate beam significantly worse (peak-to-peak value of about $15 \mu \mathrm{K}$ ). This reduction of GSC from the intermediate beam is due to the corresponding contribution to the integrated response from the beam at few degrees from the beam centre direction, significantly reduced in the actual optimized optical design ${ }^{16}$.

\footnotetext{
${ }^{16}$ In fact, the (per cent) fractional contribution, $f_{\%}$, is now $\simeq 0.045$ (0.39) for the intermediate (far) beam, while it was about 0.6 (1) for the intermediate (far) beam in the case of the previous analysis.
} 
C. Burigana et al.: Trade-off between angular resolution and straylight contamination. II., Online Material p 3
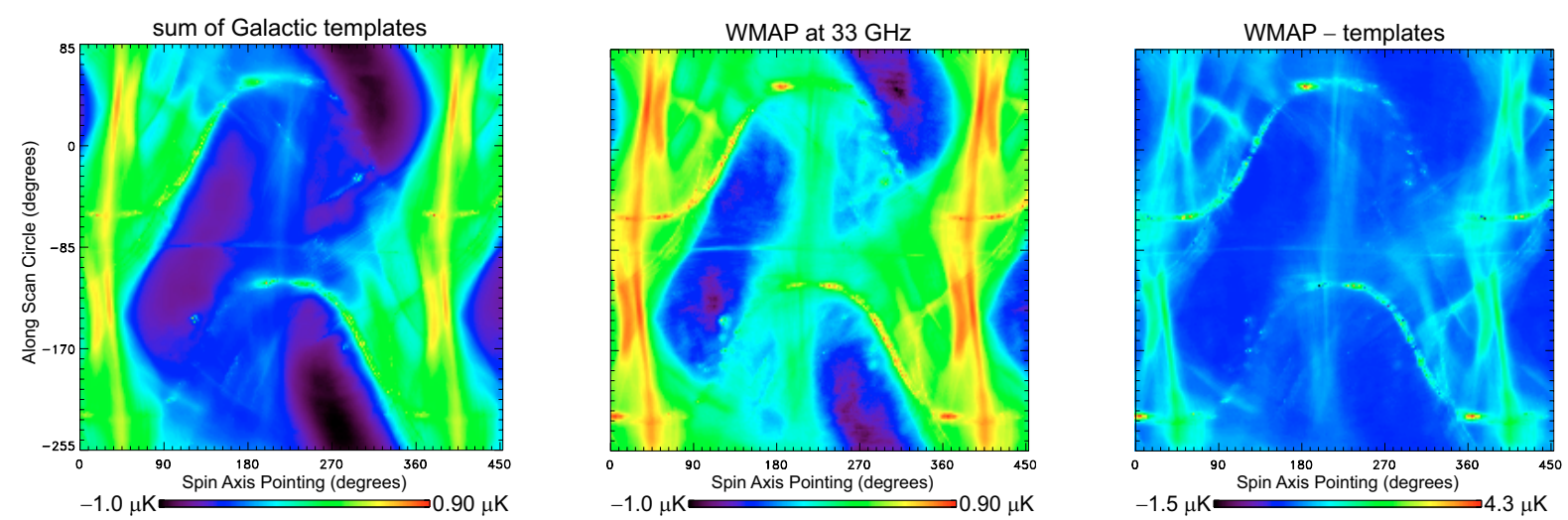

Fig. B.1. The same as in Fig. 2, but for the simulation at $30 \mathrm{GHz}$ and the overall straylight signal. For a better comparison the adopted temperature range is the same in the two panels, although the minimum and maximum values are just different, as it is evident from the different peak-to-peak values reported in Table B.1. The right panel reports (antenna temperature in linear, not logarithmic, scale, in this case) the difference between the middle panel and the left panel (see also the text).

Table B.1. The same as in Table 1, but for the beam simulated at $30 \mathrm{GHz}$. We also report the straylight contamination from the free-free emission as derived from the $\mathrm{H} \alpha$ map and the combined straylight from this free-free emission template and dust emission without assuming correlation between dust and free-free emission. Finally, we report the result based on the WMAP map at $33 \mathrm{GHz}$, including all components.

\begin{tabular}{|c|c|c|c|c|c|c|}
\hline \multicolumn{7}{|c|}{ LFI27 SWE } \\
\hline Beam & & & & & Skewness & Kurtosis \\
\hline Region & Average & Variance & rms & Peak-to-peak & Index & Index \\
\hline \multicolumn{7}{|c|}{ Dust + "correlated" diffuse free-free emission } \\
\hline $\mathrm{I} 12$ & $3.13 \times 10^{-2}$ & $1.15 \times 10^{-2}$ & $1.07 \times 10^{-1}$ & $2.72 \times 10^{+0}$ & $9.45 \times 10^{+0}$ & $1.21 \times 10^{+2}$ \\
\hline F12 & $4.75 \times 10^{-1}$ & $2.62 \times 10^{-1}$ & $5.12 \times 10^{-1}$ & $3.09 \times 10^{+0}$ & $1.64 \times 10^{+0}$ & $2.32 \times 10^{+0}$ \\
\hline $\mathrm{I}+\mathrm{F}$ & $5.07 \times 10^{-1}$ & $2.71 \times 10^{-1}$ & $5.20 \times 10^{-1}$ & $3.09 \times 10^{+0}$ & $1.53 \times 10^{+0}$ & $1.90 \times 10^{+0}$ \\
\hline \multicolumn{7}{|c|}{ Diffuse synchrotron emission } \\
\hline $\mathrm{I} 12$ & $2.97 \times 10^{-2}$ & $2.16 \times 10^{-3}$ & $4.64 \times 10^{-2}$ & $7.22 \times 10^{-1}$ & $6.72 \times 10^{+0}$ & $5.89 \times 10^{+1}$ \\
\hline F12 & $4.26 \times 10^{-1}$ & $9.94 \times 10^{-2}$ & $3.15 \times 10^{-1}$ & $1.47 \times 10^{+0}$ & $1.27 \times 10^{+0}$ & $7.35 \times 10^{-1}$ \\
\hline $\mathrm{I}+\mathrm{F}$ & $4.56 \times 10^{-1}$ & $1.01 \times 10^{-1}$ & $3.17 \times 10^{-1}$ & $1.46 \times 10^{+0}$ & $1.18 \times 10^{+0}$ & $4.90 \times 10^{-1}$ \\
\hline \multicolumn{7}{|c|}{ H II regions } \\
\hline $\mathrm{I} 12$ & $4.33 \times 10^{-3}$ & $2.54 \times 10^{-3}$ & $5.04 \times 10^{-2}$ & $4.65 \times 10^{+0}$ & $4.27 \times 10^{+1}$ & $2.91 \times 10^{+3}$ \\
\hline F12 & $5.79 \times 10^{-2}$ & $5.78 \times 10^{-3}$ & $7.60 \times 10^{-2}$ & $7.61 \times 10^{-1}$ & $1.80 \times 10^{+0}$ & $3.73 \times 10^{+0}$ \\
\hline $\mathrm{I}+\mathrm{F}$ & $6.23 \times 10^{-2}$ & $8.40 \times 10^{-3}$ & $9.17 \times 10^{-2}$ & $4.84 \times 10^{+0}$ & $8.41 \times 10^{+0}$ & $2.84 \times 10^{+2}$ \\
\hline \multicolumn{7}{|c|}{ Sum of the above components } \\
\hline $\mathrm{I} 12$ & $6.54 \times 10^{-2}$ & $3.18 \times 10^{-2}$ & $1.78 \times 10^{-1}$ & $5.34 \times 10^{+0}$ & $9.35 \times 10^{+0}$ & $1.25 \times 10^{+2}$ \\
\hline F12 & $9.60 \times 10^{-1}$ & $8.02 \times 10^{-1}$ & $8.96 \times 10^{-1}$ & $5.05 \times 10^{+0}$ & $1.51 \times 10^{+0}$ & $1.74 \times 10^{+0}$ \\
\hline $\mathrm{I}+\mathrm{F}$ & $1.03 \times 10^{+0}$ & $8.28 \times 10^{-1}$ & $9.10 \times 10^{-1}$ & $7.77 \times 10^{+0}$ & $1.42 \times 10^{+0}$ & $1.46 \times 10^{+0}$ \\
\hline \multicolumn{7}{|c|}{ Diffuse free-free emission from $\mathrm{H} \alpha$ map } \\
\hline $\mathrm{I} 12$ & $2.34 \times 10^{-2}$ & $3.35 \times 10^{-3}$ & $5.78 \times 10^{-2}$ & $1.76 \times 10^{+0}$ & $7.26 \times 10^{+0}$ & $1.01 \times 10^{+2}$ \\
\hline F12 & $1.88 \times 10^{-1}$ & $1.55 \times 10^{-2}$ & $1.24 \times 10^{-1}$ & $5.75 \times 10^{-1}$ & $6.51 \times 10^{-1}$ & $-2.35 \times 10^{-1}$ \\
\hline $\mathrm{I}+\mathrm{F}$ & $2.11 \times 10^{-1}$ & $2.13 \times 10^{-2}$ & $1.46 \times 10^{-1}$ & $2.15 \times 10^{+0}$ & $1.31 \times 10^{+0}$ & $4.55 \times 10^{+0}$ \\
\hline \multicolumn{7}{|c|}{ Dust + diffuse free-free emission from $\mathrm{H} \alpha$ map } \\
\hline I12 & $2.42 \times 10^{-2}$ & $3.49 \times 10^{-3}$ & $5.91 \times 10^{-2}$ & $1.78 \times 10^{+0}$ & $7.15 \times 10^{+0}$ & $98.5 \times 10^{+1}$ \\
\hline F12 & $1.99 \times 10^{-1}$ & $1.78 \times 10^{-2}$ & $1.33 \times 10^{-1}$ & $6.30 \times 10^{-1}$ & $6.78 \times 10^{-1}$ & $-1.85 \times 10^{-1}$ \\
\hline $\mathrm{I}+\mathrm{F}$ & $2.23 \times 10^{-1}$ & $2.37 \times 10^{-2}$ & $1.54 \times 10^{-1}$ & $2.18 \times 10^{+0}$ & $1.23 \times 10^{+0}$ & $3.80 \times 10^{+0}$ \\
\hline \multicolumn{7}{|c|}{ WMAP at $33 \mathrm{GHz}$} \\
\hline $\mathrm{I} 12$ & $1.10 \times 10^{-1}$ & $1.05 \times 10^{-1}$ & $3.24 \times 10^{-1}$ & $6.17 \times 10^{+0}$ & $7.75 \times 10^{+0}$ & $7.72 \times 10^{+1}$ \\
\hline F12 & $1.37 \times 10^{+0}$ & $1.51 \times 10^{+0}$ & $1.23 \times 10^{+0}$ & $6.76 \times 10^{+0}$ & $1.40 \times 10^{+0}$ & $1.45 \times 10^{+0}$ \\
\hline $\mathrm{I}+\mathrm{F}$ & $1.48 \times 10^{+0}$ & $1.61 \times 10^{+0}$ & $1.27 \times 10^{+0}$ & $7.18 \times 10^{+0}$ & $1.29 \times 10^{+0}$ & $1.07 \times 10^{+0}$ \\
\hline
\end{tabular}

